\title{
Structural and Dynamic Properties of Hydrophobic Eutectic Solvents Based on Menthol and Fatty Acids: a Molecular Dynamics Simulation Study
}

\section{Samaneh Barani Pour}

Azarbaijan Shahid Madani University

Jaber Jahanbin Sardroodi ( $\sim$ jsardroodi@azaruniv.ac.ir )

Azarbaijan Shahid Madani University

Alireza Rastkar Ebrahimzadeh

Azarbaijan Shahid Madani University

\section{Research Article}

Keywords: Hydrophobic Eutectic Mixtures, Menthol, Fatty acids, Molecular dynamic simulation,

Dynamical and Structural Properties, Angular Distribution Functions (ADFs), Self-Diffusion Coefficients

Posted Date: December 1st, 2021

DOI: https://doi.org/10.21203/rs.3.rs-1083302/v1

License: (c) (i) This work is licensed under a Creative Commons Attribution 4.0 International License.

Read Full License 


\title{
Structural and dynamic properties of Hydrophobic Eutectic Solvents Based On Menthol and Fatty Acids: A Molecular Dynamics Simulation Study
}

\author{
Samaneh Barani Pour ${ }^{a, b, d}$, Jaber Jahanbin Sardroodi ${ }^{a, b, d *}$, Alireza Rastkar Ebrahimzadeh ${ }^{a,}$ \\ $c, d$ \\ ${ }^{a}$ Molecular Simulation Lab, Azarbaijan Shahid Madani University, Tabriz, Iran \\ ${ }^{b}$ Department of Chemistry, Azarbaijan Shahid Madani University, Tabriz, Iran \\ 'Department of Physics, Azarbaijan Shahid Madani University, Tabriz, Iran \\ ${ }^{\mathrm{d}}$ Molecular Science and Engineering Research Group (MSERG), Azarbaijan Shahid \\ Madani University, Tabriz, Iran \\ *Corresponding Author E-mail: jsardroodi@azaruniv.ac.ir
}

\begin{abstract}
The structural and dynamical properties of the binary mixture of Menthol (MEN) and Fatty acids (FAs) were investigated using molecular dynamics simulations. We focused on the relationship between the structural and dynamical properties of the eutectic mixtures of MEN and FAs with different molar percentages of FAs. Structural properties of the eutectic mixtures were characterized by calculating the combined distribution functions(CDFs), the radial distribution functions (RDFs), and the angular distribution functions (ADFs), and the Hydrogen bonding network between species and Spatial distribution functions (SDF). Further interaction between menthol and Caprylic acid molecules was confirmed by the results of these analyzes. Also, the transport properties of the mixtures were investigated by using the mean square displacement (MSD) of the centers of mass of the species, self-diffusion coefficients
\end{abstract}


and vector reorientation dynamics (VRD) of bonds. The simulation results indicated that intermolecular interactions have a significant effect on the dynamic properties of species.

Keywords: Hydrophobic Eutectic Mixtures, Menthol, Fatty acids, Molecular dynamic simulation, Dynamical and Structural Properties, Angular Distribution Functions (ADFs), Self-Diffusion Coefficients.

\section{INTRODUCTION}

Menthol [1-meMENl-4-(1-meMENleMENl) cyclohexan-3-ol] is cyclic terpene alcohol that may be extracted from the oil of plants of the Mentha genus[1].This natural product has been used for medicinal purposes, cosmetics, as a flavor and, as an intermediary in the production of widely used industrial solvents (deep eutectic solvents)[2]. The deep eutectic solvents (DESs) are made by strong interactions between the Hydrogen bond donor (HBA) and Hydrogen bond acceptor (HBD)[3]. Eutectic mixtures have a melting point much lower than their individual components. DESs were first introduced by Abbot et al. (2003) for a mixture of choline chloride and urea[4]. It has been found that DESs and ILs have similar physical characteristics such as viscosity, conductivity, and surface tension [5]. DESs are a good alternative to ILs due to their biodegradability, lower toxicity, and reasonable price[6]. The various applications of eutectic solvents in chemical and pharmaceuticals make the use of DESs very appealing. So far, the most widely proposed solvents in the open literature are hydrophilic eutectic solvents thus are unstable in water. In 2015, Hydrophobic DES was receiving a great amount of attention due to the stable hydrophobic phase in contact with water and inexpensive and natural components that follows the principles of green chemistry [5]. Reports showed that the hydrophobic DESs based on ammonium salts and Decanoic acid were able to recovery of volatile fatty acids from aqueous solutions [7]. Marrucho et al. have reported several 
Hydrophobic deep eutectic solvents composed of natural, nontoxic compounds, i.e., terpenes and fatty acids [8]. Eutectic mixtures of menthol and saturated fatty acids derived from coconut oil and palm kernel oil [9]. Some DESs have found very interesting applications in the extraction of biomolecules and indium from aqueous solutions [8]. Because eutectic solvents have been widely used in the isolation of pollutants from aqueous solutions, predicting thermos-physical and structural properties is particularly important. MD simulation may provide molecular insight into the eutectic mixtures for medicinal chemists. In the present study, we were interested in focusing on the effect of the percentage composition of species and temperature on the dynamic and structural properties of the eutectic solvents based on menthol and fatty acid. Binary mixtures of Caprylic Acid: Menthol, Decanoic acid: Menthol, and Lauric Acid: Menthol were prepared. Investigation of structural and dynamic properties of eutectic solvents can provide a valuable perspective in the design of these solvents.

\section{COMPUTATIONAL METHODS}

The NAMD 2.13 software package was used to run all MD simulations [10]. Three fatty acids, Caprylic acid (CAP), Decanoic acid (DEC), and Lauric acid (LUA), and menthol, were selected. Packmol software package was used for the preparation of the eutectic mixtures with the different molar percentages of FAs [11]. The information about the simulated boxes and their different combinations (HBD and HBA) with abbreviations are listed in Table 1. The initial simulation box included with the mole percent of FAs at $20.0 \%$ and $80.0 \%$ of MEN was provided using distributed randomly. In the next step, the number of FAS molecules was increased for the preparation of the mixtures with the mole percent of FAs at $70.0 \%$. First, the initial configurations were minimized to remove bad contacts followed. The binary mixtures with the most intermolecular interaction between HBD and HBA were selected and their 
temperature was increased slowly from 0 to $353 \mathrm{~K}$ and then decreased back to $323 \mathrm{~K}$ and 300 $\mathrm{K}$ with a temperature gradient of $1 \mathrm{~K} / \mathrm{FS}$. The Nose-Hoover thermostat [12] and the ParrinelloRahman pressure coupling [13] were used to maintain a constant temperature and pressure, respectively, during the simulation. Finally, the binary mixtures were equilibrated for $50 \mathrm{~ns}$ in the NPT ensemble with a time-step of $1 \mathrm{fs}$. The particles mesh Ewald (PME) method and the Lennard-Jones (LJ) 6-12 potential with a cut-off distance of $12 \mathrm{~A}^{\circ}$ were applied for long-range and short-range interactions, respectively.

Table 1. Names of the simulated eutectic solvents and their different combinations (HBD and HBA) with abbreviations, and the MEN: FAs ratios in the binary mixtures.

\begin{tabular}{lllll}
\hline Name & HBD & HBA & \%FAs & n $_{\text {MEN }} \mathbf{n}_{\text {FAs }}$ \\
\hline MCA & Caprylic acid (CAP) & Menthol (MEN) & $20,44,70$ & $800: 200,560: 440,300: 700$ \\
MDA & Decanoic acid (DEC) & Menthol & $20,35,70$ & $800: 200,650: 350,300: 700$ \\
MLA & Lauric acid (LUA) & Menthol & $20,25,70$ & $800: 200,750: 250,300: 700$ \\
\hline
\end{tabular}



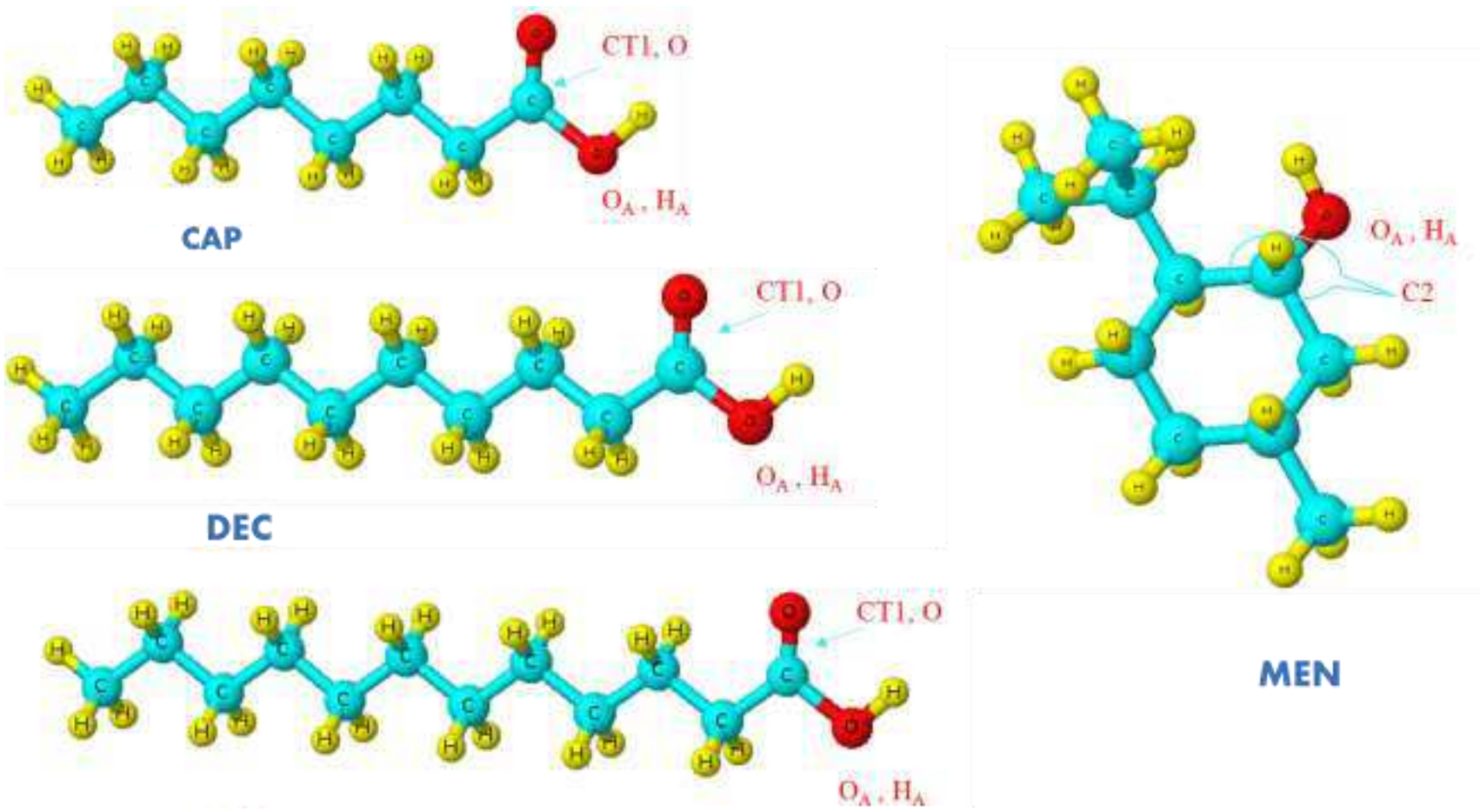

MEN

LUA

Fig. 1. Schematic of Menthol (MEN) and Fatty acids (FAs) with the main labels.

\section{RESULTS AND DISCUSSION}

\subsection{Radial Distribution Functions (RDFs)}

Radial distribution functions (RDFs) were computed to characterize the various interactions between species in the binary mixtures of MEN and FAs. To better understand the structural properties of the binary mixtures, RDFs of the $\mathrm{H}$ atoms of HBD around the $\mathrm{O}$ atoms of the HBA were obtained. Fig. 1 depicts labels of the atoms in the hydrogen bond donor and acceptor. The FAs -- MEN RDFs have a main peak and a small plateau in the vicinity of the first maximum peak, while all MEN -- MEN RDFs have a similar shape with two maxima. The sharp peaks of HA FAs - OA MEN RDFs are a clear indication of the interaction between the hydroxyl hydrogen of MEN and the O atoms of FAs at a distance of $2 \AA$ (see Fig 2a). 
The pair interaction of two species has more intensity in the binary mixtures of MEN and with $\% \mathrm{CAP}=44, \% \mathrm{DEC}=35$, and $\%$ LUA $=25$ (see Fig. $2 \mathrm{~b}$ and Fig. $2 \mathrm{c}$ ). The MEN -- MEN RDF was investigated in the binary mixtures with different percentages of FAs molecules. The DEC concentration dependence of MEN -- MEN RDFs is shown in Figure 2d. The shoulder and the main peak are located at about 3 and $2 \mathrm{~A}^{\circ}$ in the binary mixtures of DEC and MEN. The height of the first peak of MEN -- MEN RDF gradually was decreased with increasing the mole percent of FAs. These results indicate that the probability distribution of menthol molecules around FAs molecules increases in the binary mixtures. In order to the influence of the temperature, the site-site RDFs between the CTn-CTn tails $(n=2,4,8$, and 12 corresponding to the number of carbon atoms of the alkyl chain of FAs) and the carboxylic head of FAs with the OA atom of MEN and were analyzed at different temperatures (see Fig.3). The site-site RDFs of the carbon atoms of FAs around the OA atom of MEN can be seen in Fig. 2e. The position of the maximum peaks of RDFs between the $\mathrm{C}$ atoms of the alkyl chain of the OA atom of MEN is located compared to RDFs between the carboxylic head of FAs and the OA atom of MEN at farther distances. There is a strong interaction between the HA atom of FAs and the OA atom of MEN at $300 \mathrm{~K}$. For distances more than $6 \mathrm{~A}^{\circ}$, the height of the first peak of the RDFs almost no changes at different temperatures. The distribution of the $\mathrm{C}$ atoms of FAs in the nonpolar region around the OA atom of MEN is obviously different at different temperatures. RDFs between the CTn - CTn tails and the OA atom of MEN indicate that the CT1- CT1 aggregation degree around the OA atom of MEN is reduced with increasing temperature. The coordination number of the RDFs peaks at temperature $353 \mathrm{~K}$ is less commonly seen than the $\mathrm{CN}$ of the RDFs at temperature $300 \mathrm{~K}$ because of the reorientation dynamics of the bond vectors. 


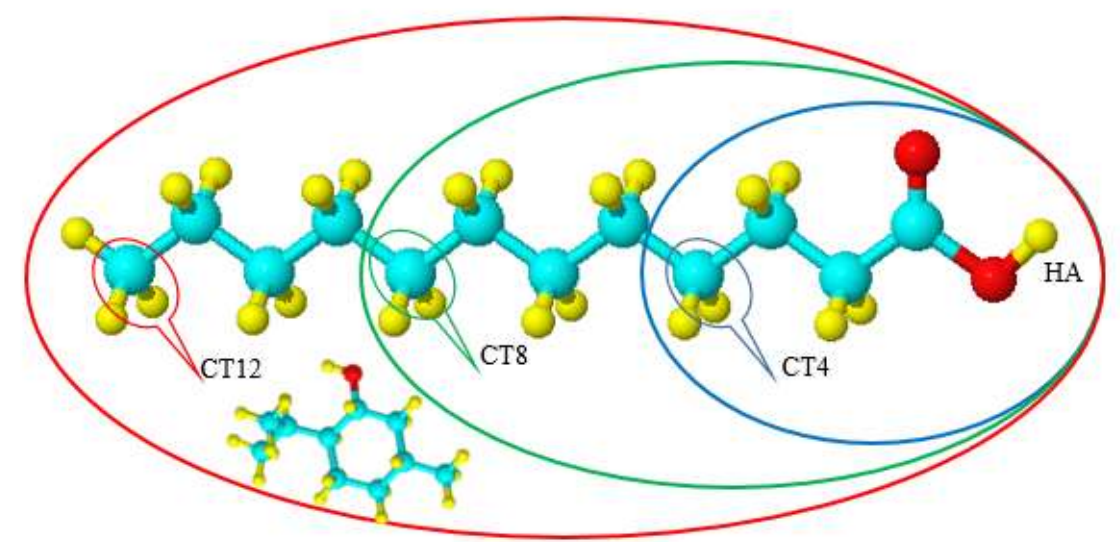

Fig 3. The regions of the LUA molecule in the binary mixture: CT4 (blue ellipse), CT8 (green ellipse), and CT12 (red ellipse).

\subsubsection{Coordination number}

The coordination number is defined as the number of nearest neighbours of a central molecule in the mixture [14]. Generally, the $\mathrm{CN}$ can be gained by integrating the surface region surrounded at the first solvation shell (rfs). The CNs are calculated by Equation (1).

$N=4 \pi \rho \int_{0}^{r_{\text {sell }}} r^{2} g(r) d r$

, where $\rho$ and $r_{\text {sell }}$ represent the number density and the first minimum in the RDFs[15]. Coordination numbers of the molecules around each other in the binary mixtures are listed in Table S1. It seems that the first solvation shell of the MEN -- MEN RDF can accommodate a fewer number of menthol molecules in the binary mixtures of MCA, MDA, and MLA with $\% \mathrm{FAs}=44,35$, and 25 , respectively 
Decreasing the aggregation degree of the MEN molecules around each other can be attributed to the strong interactions between the two species in the binary mixtures. Most likely, the FAs have been acted as hydrogen-bond acceptors and hydrogen-bond donors in the binary mixtures. Dual role as both HBA and HBD of acids in the binary mixtures with $\% \mathrm{FAs}=70$ is noticeably higher than other ratios. To further study temperature dependence, the site-site RDFs between the LUA and the OA atom of MEN were calculated at different temperatures. The CNs of the carbon atom of the alkyl chain (CTn - CTn) are shown in Fig. 4. In general, the CNs are reduced with increasing temperature. The coordination number of CT12 around menthol was notably greater than the CT4 in the binary mixtures of MLA. This aggregation is the result of the van der Waals force and It can be also due to very few hydrogen bonds. The competition between electrostatic and van der Waals interactions leads to the aggregation via the carbon atom (CTn) of the tail.

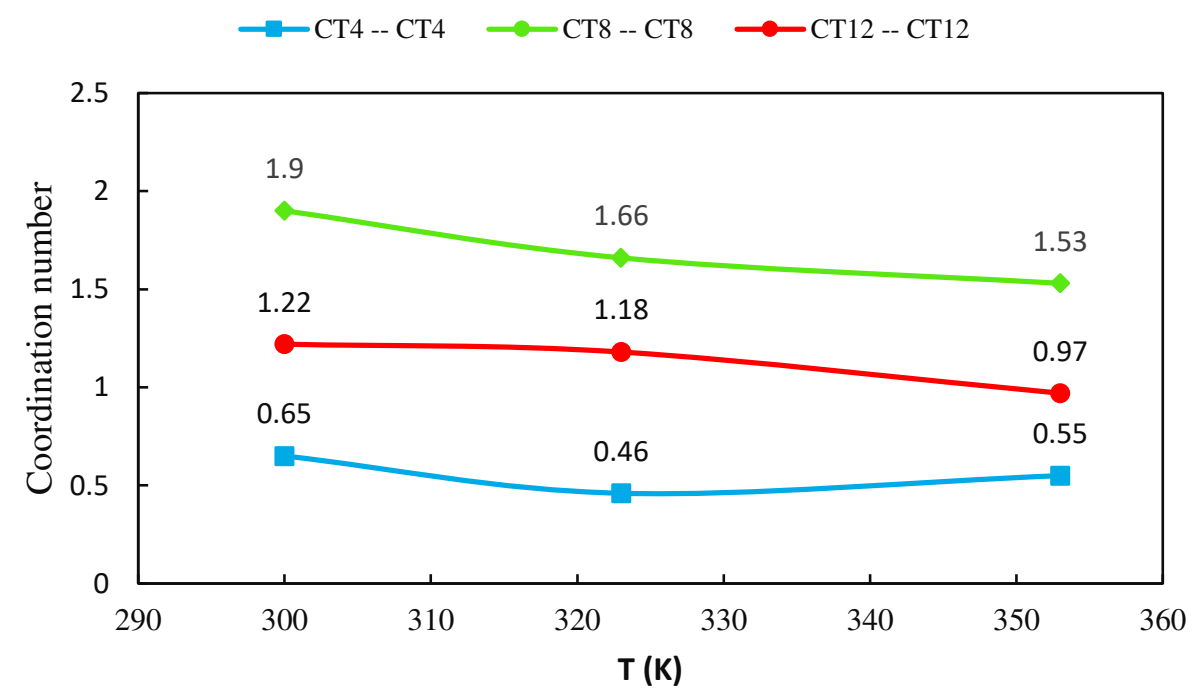

Fig. 4. Coordination numbers of tails CTn - CTn around the OA atom of MEN in the binary mixtures of LUA and MEN. 


\subsection{Hydrogen bond analysis}

In the DES, strong D-H $\cdots$ A hydrogen bonding leads to a reduction in the melting point of the eutectic mixtures. Hydrogen bond criteria have defined a distance and angle. Statistically, the probability of the observed exact value of the distance and angle is zero. According to the suggestions of Wernet et al. the set of two separate criteria (one distance and one angle) from the specific region of CDF plots were selected to determine the presence of a (strong) H-bond [16]. The selected distances and angles are given below: $2 \AA\left(150^{\circ}\right)$ for $\mathrm{HA}_{\mathrm{MEN}}-\mathrm{O}_{\mathrm{CAP}}, 2 \AA$ $\left(135^{\circ}\right)$ for $\mathrm{HA}_{\mathrm{CAP}}-\mathrm{O}_{\mathrm{CAP}}$ and $2.25 \AA\left(150^{\circ}\right)$ for HA $\mathrm{MEN}-\mathrm{OA}_{\text {MEN }}$ pairs (see Fig. 8a, Fig. 8d and Fig. 8e). Menthol molecule with the atom labeling scheme is given in Figure S1.

The stability of the H-bonds interactions between the different atoms of MEN and the $\mathrm{O}$ atom of FAs was investigated in the binary mixture. The hydrogen-bonded network between the HA atom of MEN and the OA atom of FAs is more stable than other atoms of MEN.

The number of H-bonds between different species (HBD and HBA) was measured over time. The average number of hydrogen bonds is obtained vis the fitting of Eq. (2)

$$
F(X)=\frac{a}{\sigma \sqrt{2 \pi}} \exp \frac{-(X-\bar{X})^{2}}{2 \sigma^{2}}
$$

, where $\sigma$, and $\bar{X}$ are the standard deviation and the average number of hydrogen bonds, respectively[17]. The average number of hydrogen bonds between species is an important parameter for the formation of eutectic solvent that changes under the influence of the relative percentage of species and temperature. The hydrogen bonds in the binary mixtures in the binary mixtures of MCA, MDA, and MLA with $\% \mathrm{FAs}=44,35$, and 25 are more than hydrogen bonds in the mixtures with other ratios (see Table S2). 
The H-bond number between FAs and MEN was reduced in the binary mixtures with a higher percentage of FAs. Most likely, Decreasing the average number of hydrogen bonds is due to the distribution of the number of hydrogen bonds between the carboxyl groups of FAs (see Fig. 5a). The hydrogen-bonding network is directly affected by the temperature of the mixture. Investigation of the hydrogen bonding network in the binary mixture at the temperature range of 300 to 353 also showed that the H-bond number was slightly decreased with raising the temperature (see Fig. 5b). The total time that a hydrogen-bond network has established between HBD and HBA is described using the percent occupancy [18]. This analysis has depicted the stability and persistence of hydrogen bonds between the possible HBD and HBA pairs [19]. The stabilization of the hydrogen bonding network between the different species is provided in Figure $6 \mathrm{a}-6 \mathrm{~b}$. Figure 1 shows the Hydrogen bond percent occupancies of the HA atoms of MEN and the $\mathrm{O}$ atom FAs in the binary mixture with a molar percentage of $70 \%$ at $323 \mathrm{~K}$. The percent occupancy of the hydrogen bonding with FAs was the highest in the binary mixture of MCA. The stability of the H-bond between the two species was decreased with increasing carbon chain length of FAs from C8 to C12. The occupancy of the hydrogen bonding between three kinds of HBA and MEN has the highest content in the binary mixtures of MCA, MDA, and MLA with $\% \mathrm{FAs}=44,35$, and 25, respectively. This order corresponds to the number of intermolecular hydrogen bonding in the binary mixture. The strong interaction between FAs and MEN at the temperature of $300 \mathrm{~K}$ is probably due to the hydrogen bonding with the highest percent occupancies.

\subsection{Spatial Distribution Functions (SDFs)}

For further visualization of the local structure of the eutectic mixture, spatial distribution functions (SDFs) were analyzed [20]. SDFs can present the desirable sites for intermolecular hydrogen-bond interactions. Three-dimensional spatial distribution functions of different species around a reference molecule were plotted in Figure 7 (a, b, c, d, e, f). The distribution 
of menthol molecules around the Carboxylic acid group of FAs is seen vividly from the isosurfaces Fig7 (a, b and c). The density distribution function (Dens) of the HA atom of MEN around the OA atom of FAs was investigated in the binary mixtures. The values obtained for Dens are given in Table 3. The values of dens were determined from the first minimum of the peak of the corresponding particle density. The dens between the HA atom of MEN and the OA atom of CAP are $0.25,0.19$, and 0.15 in the binary mixtures with $\% \mathrm{FAs}=44,35$, and 25 , respectively. The maximum $\mathrm{H}$-bond of the two species around each other of approximately 201.21 was observed in the binary mixtures with $\%$ CAP $=44$. Therefore, the results were confirmed using H-bond analysis. These observations are in agreement with the results of the combined distribution function and Hydrogen bond analysis. Furthermore, the probabilities of the FAs molecules around the hydroxyl group of MEN increase with the number of acid molecules in the simulation box (see Fig.7 (d, e, f)). SDFs of acid molecules around each other showed that these molecules are mainly distributed around each other in $\mathrm{H}-\mathrm{O}$ and $\mathrm{O}=\mathrm{C}$ sites of the carboxyl group. In the mole percent of FAs at 70\%, the reduction of the distribution of the two species around each other in the binary mixtures is more likely related to the accumulation of acid molecules around each other. Increasing temperatures cause a shift in the aggregation behavior of molecules around each other. This result may be related to the local solvation structure in the binary mixtures. The maximum distribution between the two species of MEN and FAs is not generally the same at different temperatures. It should be noted, the isovalues were selected the reflect the completion of the first solvation shell. [21]. 


\begin{tabular}{|c|c|c|c|}
\hline & \\
\hline & & \\
\hline & & \\
\hline
\end{tabular}

Figure 7. Spatial Distribution functions (SDFs) of the binary mixtures with the mole percent of FAs at $70 \%$ at $323 \mathrm{~K}$. Green isosurfaces correspond to LUA, yellow isosurfaces correspond to DEC, blue isosurfaces are CAP molecules, pink isosurfaces are MEN molecules. The reference molecules are Caprylic acid (a-c), or MEN molecule (d-f).

\subsection{Tracing [MEN] [FAs] H-bonds Using Combined Distribution Function (CDF)}

The distance criteria of $\mathrm{H}$-bond in the binary mixtures was determined from the first minimum of the peaks of the corresponding RDFs. However, the distance criteria may not be adequate to capture the presence of a strong H-bond in the binary mixtures [16]. Therefore, the combined radial/angular distribution functions were obtained for all possible pairs. The composed CDFs of the RDF between the hydroxyl hydrogen (HA) site of MEN and the OA atom of the FAs as the $\mathrm{X}$-axis, the Angular Distribution Function (ADF) between two vectors (R1 and R2) as the Y-axis are shown in Figure 8a. 
$\mathrm{H}$-bonds (weak or strong) were found at the angles range between 130 and $150^{\circ}$ and distances of less than $2 \AA$. Figure 8 a shows that there is more menthol around CAP than the other acids in the binary mixtures (see Fig.8b and Fig.8c). CDFs analysis also displays strong H-bond interaction, i.e. $-\mathrm{OH} \ldots \mathrm{O}=\mathrm{C}$ in the binary mixtures of MCA, MDA, and MLA with $\% \mathrm{FAs}=$ 44, 35, and 25, respectively. The arrangement of the neighbour MEN molecules is perpendicular $\left(130^{\circ}<\theta<160^{\circ}\right)$ and located a distances of 2 and $3 \AA$ from the reference MEN molecule. In the binary mixtures with the same mole percent of FAs, the presence of CAP was greatly inhibited strong interactions between MEN molecules compared to other FAs.

For further intuition about the bridging role of species in binary mixtures, Respective distance/distance CDFs including three neighbor molecules (MEN $\cdots$ FAs $\cdots$ MEN) were computed. The possibility of connecting two MEN molecules as the HBD by one [FAs] bridge was investigated in the binary mixtures with the different mole percent of FAs. The respective CDF includes the two axes that the $\mathrm{X}$-axis illustrates the distance between the $\mathrm{H}$ atom of the MEN, HA, and carboxyl oxygen of the neighbor FAs, OA, and the Y-axis illustrates the distance of fatty acid-menthol neighbors $(\mathrm{O}$-- HA). The intense probability was observed at distances of $2 \AA$ and $3 \AA$. The results showed that the bidentate hydroxyl group of [MEN] can bind two FAs molecules in the binary mixtures (see Fig 9a-9c). The occurrence probability of the $[\mathrm{MEN}] \cdots[\mathrm{FAs}] \cdots[\mathrm{MEN}]$ structure in the binary mixtures with a molar percentage of $70 \%$ was attenuated due to the H-bonding formation between FAs molecules.

\section{Non-bonded interaction energy}

The non-bonded interaction energy is an important analysis for understanding microscopic properties in MD simulations. The non-bonded energy includes two terms, short-range van der Waals (vdW) interactions and long-range electrostatic interactions, which are calculated 
Lennard-Jones (12-6) function and Coulombic equation, respectively[22]. The vdW and Coul interactions are described by the following equations:

$$
E_{\text {total }}=E_{\text {Coulombic }}+E_{v d W}=\frac{e^{2}}{4 \pi \varepsilon_{0}} \sum_{i \neq j} \frac{q_{i} q_{j}}{r_{i j}}+\sum_{i \neq j} D_{0, i j}\left[\left(\frac{R_{0, i j}}{r i j}\right)^{12}-2\left(\frac{R_{0, i j}}{r i j}\right)^{6}\right]
$$

The values of the non-bonded energies are reported in Table S3. It could be seen from Table $\mathrm{S} 3$, Ecoul and $\mathrm{E} \mathrm{vdW}$ are $-3235.06 \mathrm{~kJ} / \mathrm{mol}$ and $-8.10 \mathrm{~kJ} / \mathrm{mol}$, respectively, in the binary mixtures CAP and MEN with $\%$ FAs $=44$ at 323 . The results show that the vdW interaction energy is predominant between HBA and HBD. The electrostatic energy values are so minimal that they can be ignored. The absolute value of the non-bonded energies shows that the interaction between LUA and MEN were significantly greater in the binary mixture with $\% \mathrm{FAs}=25$ at $323 \mathrm{~K}$ than the other ratios. In terms of energy, this percentage combination may be more suitable for the preparation of the eutectic mixture based on acid and menthol. However, interactions between two molecules show that temperature can be a more effective factor in the interaction. Investigation of the interaction energy of MEN: MEN in the binary mixture with $\%$ FAs $=44$ showed that the amount of energy was slightly decreased under various temperatures from 300 to $353 \mathrm{~K}$ (see Fig. 10). According to Figure S1, increasing the length of the alkyl chain of FAs may prevent further interaction between the HBA and HBD.

\section{Dynamical and transport properties}

The mean-squared displacements (MSDs) of species were measured to describe the migration of species [23]. The mean square displacements (MSDs) of species as a function of time were plotted for binary mixtures of FAs and MEN in the same ratio in Figure 11a-11b. The low slope of the MSD curve of menthol molecules indicates a strong interaction between the two components in the binary mixture of menthol and Caprylic acid compared to the mixture of MDA. The self-diffusivity coefficient is the macroscopic dynamical property which is 
calculated from MD simulation [24]. The self-diffusion coefficients of species were obtained from the slopes of the lines fitted to the MSD curves using the Einstein relation:

$D=\lim _{t \rightarrow \infty}\left\langle|\Delta r(t)|^{2}\right\rangle / 6 t$

$\left\langle\Delta|r(t)|^{2}\right\rangle=\frac{1}{N}\left\langle\sum_{i=1}^{N}\left|r_{i}^{C O M}(t)-r_{i}^{C O M}(0)\right|^{2}\right\rangle$

Where, $\left\langle\Delta|r(t)|^{2}\right\rangle$ is the mean-square displacement (MSD) of species i. To ensure the measured values of self-diffusion coefficients, the diffusive regime was identified and sampling was performed in this regime [25]. The $\beta$ parameter was used to determine the location of the diffusive regime in the binary mixtures [26]. This parameter, $\beta$ is calculated according to:

$\beta=\frac{\mathrm{d} \log _{10}<\Delta \mathrm{r}(\mathrm{t})^{2}>}{\mathrm{d} \log _{10} \mathrm{t}}$

The reduced viscosity at higher temperatures has led to more rapid migration of species in the diffusive regime. The calculated self-diffusion coefficient values from the diffusive regime are collected in Table 2. The self-diffusion coefficients of species in the binary mixtures with a molar percentage of $70 \%$ are ranked as MDA $>$ MCA $>$ MLA. The self-diffusions of species decrease with added the acid molecules into the simulation box. It should be noted, the remarkable changes were found in the binary mixtures of MCA, MDA, and MLA with \%FAs $=44,35$, and 44 . Reducing self-diffusion can be justified by intermolecular interactions.

\subsection{Vector Reorientation Dynamics (VRD)}

The vector reorientation dynamics is a common quantity that can be determined by both experimental techniques[27] and molecular dynamics simulation methods. VRD is computed from equation (4), 
$V R D(\tau)=N \cdot\left\langle\sum_{t=0}^{T-\tau} \vec{a}_{i}(t) \cdot \vec{a}_{i}(t+\tau)\right\rangle_{i}$

, where $\vec{a}_{i}(t)$ and $\vec{a}_{i}(t+\tau)$ are a vector selected on a molecule in step $\mathrm{t}$ and at the later time $\mathrm{t}$ $+\tau$, respectively[28]. The reorientation dynamic of the bonds vector of the MEN molecules is displayed in Fig.12a. As one can see, the reorientation of the bond vectors OA-HA in MEN is slower than the other bond vectors due to intermolecular interactions. Therefore, the reorientation dynamic of HA-OA vectors is discussed in this section. For the reorientation of the HA--OA bond vectors, the blue and red solid lines and the green dashed lines indicate VRD in the binary mixtures of MCA, MDA, and MLA, respectively (see Fig. 12b). VRD of the HA-OA bond in Capric acid is considerably faster than this bond in other acids. VRD of the HA-OA bond of Capric acid in the binary mixture of MCA with $\%$ FAs $=44$ was lowest and increased at the $353 \mathrm{~K}$ (see Fig. 12c). Interactions between FAs and MEN resulted in the lowest orientation velocity in the mixture of MCA with $\% \mathrm{CAP}=44$. Similarly, the HA--OA bond has the fastest reorientation dynamic in the binary mixture with \%LUA=25 at $353 \mathrm{~K}$ compared to other temperatures. The slower orientation of the HA--OA bond can be related to the interaction of the hydrogen bond between the acid molecules that are reported in the section.

\section{Thermo-physical Properties Analysis}

The shear viscosity $\eta$ can be obtained from the xy elements of the molecular pressure tensor using the Green-Kubo expression [29]. $\eta$ is given by

$\eta=\frac{\mathrm{V}}{k_{B} T} \int_{0}^{\infty} d t<P_{x y}(0) P_{x y}(t)>$,

, where $\mathrm{V}$ and $\mathrm{T}$ are stands for the molar volume and the temperature, respectively. $\mathrm{KB}$ is the Boltzmann constant [30]. 
$P_{x y}=\sum_{i=1}^{N} \frac{p_{i}^{x} p_{i}^{y}}{m_{i}}-\sum_{i>j}^{N}\left(x_{i}-x_{j}\right) \frac{\partial u_{i j}}{\partial y_{j}}$,

Here, $\mathrm{p}_{\mathrm{i}}^{\mathrm{x}}$ and $u_{i j}$ indicate the momentum component and the interaction potential, respectively. $x_{i}$ is a component of the radius vector [31]. The measured viscosity values are collected in Table 3. The values of $\eta$ for the binary mixtures are in the sequence: 121.6 and 40.14 for the binary mixtures of MCA and MDA at a temperature of $323 \mathrm{~K}$. Based on the results of the occupancy of the hydrogen bonding, the hydrogen bond network in the binary mixture of CAP and MEN is more stable than in the binary mixture of MDA. It can be concluded that the interactions between FAs and MEN molecules have an important effect on the shear viscosity of the binary mixtures. The effect of increasing FAs molecules on the shear viscosity of the binary mixtures was investigated by measuring the $\eta$ at the different molar percent of FAs. The addition of the FAs molecules into the binary mixtures increases the viscosity of all the mixtures of FAs and MEN since $\eta$ of the binary mixtures containing $44 \%$ CAP is higher $(52.451 \mathrm{mPa}$ s) than the binary mixture with the molar percentage of $20 \%(24.63$ $\mathrm{mPa} \mathrm{s}$ ). The values of the shear viscosity are in reasonable agreement with the hydrogen bonds result. The shear viscosity of the binary mixtures was decreased gradually along with the increasing temperature.

\section{CONCLUSION}

In the recent work, the correlation between the structural and dynamical properties was investigated using MD simulation. There is a strong interaction between FAs and MEN molecules in the binary mixtures of MCA, MDA, and MLA with $\%$ FAs $=44,35$, and 25, respectively. The maximum distribution between the two species of MEN and FAs in these ratios is estimated from structural analyzes such as RDF, SDF, and the density distribution 
function. The distance range of 2 to $3 \AA$ and the range of angles between 130 and $180^{\circ}$ shows the maximum number of hydrogens bonds between the two species. A single fatty acid molecule can participate as a bridge in the binary mixture because it can accept H-bonds using the $-\mathrm{COOH}$ group. The increased temperatures lead to an increase in the migration of molecules, which is probably attributed to the change in intermolecular interactions. It was also observed that the interaction between two species was reduced with the increase in length of alkyl chain length.

\section{REFERENCE}

[1] Patel, T., Ishiuji, Y., Yosipovitch, G. Menthol: a refreshing look at this ancient compound. Journal of the American Academy of Dermatology. 2007, 57, 873-8.

[2] A Farco, J., Grundmann, O. Menthol-pharmacology of an important naturally medicinal "cool". Mini reviews in medicinal chemistry. 2013, 13, 124-31.

[3] Hayyan, M., Looi, C.Y., Hayyan, A., Wong, W.F., Hashim, M.A. In vitro and in vivo toxicity profiling of ammonium-based deep eutectic solvents. PloS one. 2015, 10, e0117934. [4] Abbott, A.P., Capper, G., Davies, D.L., Rasheed, R.K., Tambyrajah, V. Novel solvent properties of choline chloride/urea mixtures. Chemical communications. 2003, 70-1.

[5] Alioui, O., Benguerba, Y., Alnashef, I.M. Investigation of the CO2-solubility in deep eutectic solvents using COSMO-RS and molecular dynamics methods. Journal of Molecular Liquids. 2020, 307, 113005.

[6] Petkovic, M., Ferguson, J.L., Gunaratne, H.N., Ferreira, R., Leitao, M.C., Seddon, K.R., et al. Novel biocompatible cholinium-based ionic liquids- toxicity and biodegradability. Green Chemistry. 2010, 12, 643-9.

[7] Florindo, C., Branco, L., Marrucho, I. Development of hydrophobic deep eutectic solvents for extraction of pesticides from aqueous environments. Fluid Phase Equilibria. 2017, 448, $135-42$. 
[8] Ribeiro, B.D., Florindo, C., Iff, L.C., Coelho, M.A., Marrucho, I.M. Menthol-based eutectic mixtures: hydrophobic low viscosity solvents. ACS Sustainable Chemistry \& Engineering. 2015, 3, 2469-77.

[9] Yeong, S.K., Idris, Z., Hassan, H.A. Palm oleochemicals in non-food applications. In: Palm Oil, Elsevier, 2012, pp. 587-624.

[10] Phillips, J.C., Braun, R., Wang, W., Gumbart, J., Tajkhorshid, E., Villa, E., et al. Scalable molecular dynamics with NAMD. Journal of computational chemistry. 2005, 26, 1781-802.

[11] Martínez, L., Andrade, R., Birgin, E.G., Martínez, J.M. PACKMOL: a package for building initial configurations for molecular dynamics simulations. Journal of computational chemistry. 2009, 30, 2157-64.

[12] Wong, K., Krishnan, R.P., Chen, C., Du, Q., Yu, D., Lu, Z., et al. The role of localgeometrical-orders on the growth of dynamic-length-scales in glass-forming liquids.

Scientific reports. 2018, 8, 1-7.

[13] Parrinello, M., Rahman, A. Polymorphic transitions in single crystals: A new molecular dynamics method. Journal of Applied physics. 1981, 52, 7182-90.

[14] Cieplak, M., Robbins, M.O. Nanoindentation of 35 virus capsids in a molecular model: relating mechanical properties to structure. PloS one. 2013, 8, e63640.

[15] Chen, T., Long, W., Zhang, C., Liu, S., Zhao, L., Hamaker, B.R. Fiber-utilizing capacity varies in Prevotella-versus Bacteroides-dominated gut microbiota. Scientific reports. 2017, 7, $1-7$.

[16] Wernet, P., Nordlund, D., Bergmann, U., Cavalleri, M., Odelius, M., Ogasawara, H., et al. The structure of the first coordination shell in liquid water. Science. 2004, 304, 995-9. 
[17] Pour, S.B., Sardroodi, J.J., Ebrahimzadeh, A.R. Structure and Dynamics of ThymolFatty Acids Based Deep Eutectic Solvent Investigated by Molecular Dynamics Simulation. Fluid Phase Equilibria. 2021, 113241.

[18] Perkins, S.L., Painter, P., Colina, C.M. Experimental and computational studies of choline chloride-based deep eutectic solvents. Journal of Chemical \& Engineering Data. $2014,59,3652-62$.

[19] Rehan Khalid, R., Tahir ul Qamar, M., Maryam, A., Ashique, A., Anwar, F., H Geesi, M., et al. Comparative Studies of the Dynamics Effects of BAY60-2770 and BAY58-2667 Binding with Human and Bacterial H-NOX Domains. Molecules. 2018, 23, 2141. [20] Reddy, T.D.N., Mallik, B.S. Protic ammonium carboxylate ionic liquids: insight into structure, dynamics and thermophysical properties by alkyl group functionalization. Physical Chemistry Chemical Physics. 2017, 19, 10358-70.

[21] Rajbangshi, J., Banerjee, S., Ghorai, P.K., Biswas, R. Cosolvent polarity dependence of solution structure in [BMIM][PF6]+ acetonitrile/1, 4-dioxane/hexane binary mixtures: Insights from composition dependent Voronoi polyhedra analyses, iso-surfaces and radial distribution functions. Journal of Molecular Liquids. 2020, 317, 113746.

[22] Seduraman, A., Klähn, M., Wu, P. Characterization of nano-domains in ionic liquids with molecular simulations. Calphad. 2009, 33, 605-13.

[23] Hashimoto, M., Yanagisawa, H., Minagawa, S., Sen, D., Ma, R., Murray, L.A., et al. TGF- $\beta$-Dependent Dendritic Cell Chemokinesis in Murine Models of Airway Disease. The Journal of Immunology. 2015, 195, 1182-90.

[24] Yeganegi, S., Sokhanvaran, V., Soltanabadi, A. Study of thermodynamic properties of imidazolium-based ionic liquids and investigation of the alkyl chain length effect by molecular dynamics simulation. Molecular Simulation. 2013, 39, 1070-8. 
[25] Kowsari, M.H., Fakhraee, M. Influence of butyl side chain elimination, tail amine functional addition, and $\mathrm{C} 2$ methylation on the dynamics and transport properties of imidazolium-based [Tf2N-] ionic liquids from molecular dynamics simulations. Journal of Chemical \& Engineering Data. 2015, 60, 551-60.

[26] Atilhan, M., Aparicio, S. Behavior of deep eutectic solvents under external electric fields: a molecular dynamics approach. The Journal of Physical Chemistry B. 2017, 121, 22132.

[27] Ponte Goncalves, A., Vyas, H. Triplet-state electron paramagnetic resonance as a probe of the molecular reorientation dynamics in doped naphthalene-tetracyanobenzene single crystals. The Journal of Chemical Physics. 1979, 70, 1560-1.

[28] Pour, S.B., Sardroodi, J.J., Ebrahimzadeh, A.R. The study of structure and interactions of glucose-based natural deep eutectic solvents by molecular dynamics simulation. Journal of Molecular Liquids. 2021, 334, 115956.

[29] Viscardy, S., Servantie, J., Gaspard, P. Transport and Helfand moments in the LennardJones fluid. I. Shear viscosity. The Journal of chemical physics. 2007, 126, 184512. [30] Nevins, D., Spera, F. Accurate computation of shear viscosity from equilibrium molecular dynamics simulations. Molecular Simulation. 2007, 33, 1261-6.

[31] Kirova, E., Norman, G. Viscosity calculations at molecular dynamics simulations. In: Journal of Physics: Conference Series, IOP Publishing, 2015, Vol. 653, p. 012106. 
Table 2: Calculated Self-Diffusion Coefficients of species at different temperatures.

\begin{tabular}{|c|c|c|c|}
\hline \multicolumn{4}{|c|}{$D \AA^{2} n s^{-1}$} \\
\hline $\mathbf{T} / \mathbf{K}$ & $\begin{array}{c}\text { MCA } \\
\text { n OCT: } n \text { MEN }\end{array}$ & CAP & MEN \\
\hline 323 & $200: 800$ & 4.29 & 5.40 \\
\hline 300 & $440: 560$ & 0.92 & 0.89 \\
\hline 323 & 440:560 & 3.25 & 3.90 \\
\hline 353 & $440: 560$ & 10.27 & 12.13 \\
\hline 323 & 700:300 & 5.34 & 6.09 \\
\hline $\mathbf{T} / \mathbf{K}$ & $\begin{array}{c}\text { MDA } \\
\text { n DEC: } n \text { MEN }\end{array}$ & DEC & MEN \\
\hline 323 & $200: 800$ & 6.35 & 11.68 \\
\hline 323 & $350: 650$ & 0.83 & 1.40 \\
\hline 323 & 700:300 & 9.15 & 19.76 \\
\hline $\mathbf{T} / \mathbf{K}$ & $\begin{array}{c}\text { MLA } \\
\text { n LUA: } n \text { LUA }\end{array}$ & LUA & MEN \\
\hline 323 & $200: 800$ & 0.83 & 1.39 \\
\hline 300 & 250: 750 & 0.095 & 0.16 \\
\hline 323 & 250: 750 & 0.60 & 1.21 \\
\hline 353 & 250: 750 & 3.63 & 7.41 \\
\hline 323 & $700: 300$ & 0.070 & 1.13 \\
\hline
\end{tabular}


Table 3. The density distribution function (Dens) of the HA atom of MEN around the OA atom of FAs in the mixtures and the shear viscosity $(\eta)$ of the binary mixtures from simulations.

\begin{tabular}{|c|c|c|c|c|}
\hline $\mathbf{T} / \mathbf{K}$ & $\begin{array}{c}\text { MCA } \\
\text { n CAP: } n \text { MEN }\end{array}$ & \multicolumn{2}{|c|}{ Dens /distance (̊̊) } & $\eta / m P a$ \\
\hline & & \multicolumn{2}{|c|}{ R--------------------------Dens } & \\
\hline 323 & $200: 800$ & 188.5 & 0.24 & 24.63 \\
\hline 300 & 440:560 & 188.5 & 0.26 & 92.13 \\
\hline 323 & 440:560 & 188.5 & 0.25 & 52.451 \\
\hline 353 & 440:560 & 188.5 & 0.2 & 15.81 \\
\hline 323 & 700:300 & 188.5 & 0.082 & 121.6 \\
\hline \multirow{2}{*}{$\mathbf{T} / \mathbf{K}$} & MDA & & & \\
\hline & n DEC: $n$ MEN & & & \\
\hline 323 & $200: 800$ & 192.5 & 0.11 & 40.14 \\
\hline 323 & 350:650 & 192.5 & 0.19 & 96.49 \\
\hline 323 & $700: 300$ & 192.5 & 0.026 & 104.51 \\
\hline \multirow{2}{*}{$\mathbf{T} / \mathbf{K}$} & MLA & & & \\
\hline & n LUA: n MEN & & & \\
\hline 323 & $200: 800$ & 197.33 & 0.12 & 216.06 \\
\hline 300 & 250: 750 & 197.33 & 0.14 & 187.98 \\
\hline 323 & 250: 750 & 197.33 & 0.15 & 80.83 \\
\hline 353 & 250: 750 & 197.33 & 0.11 & 21.480 \\
\hline 323 & 700:300 & 197.33 & 0.1 & 412.96 \\
\hline
\end{tabular}




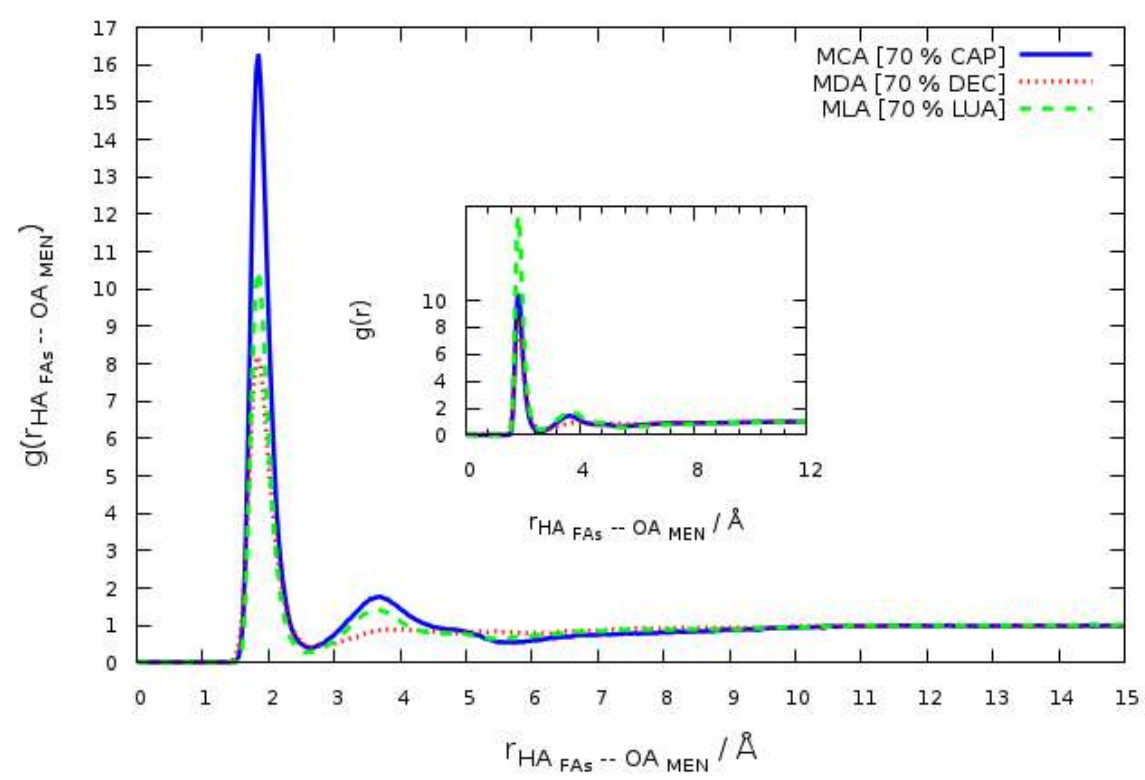

Fig. 2a. RDFs between HA atom FAs and the OA atom on the menthol molecules in the binary mixtures with $\% \mathrm{FAs}=70$ at $323 \mathrm{~K}$.

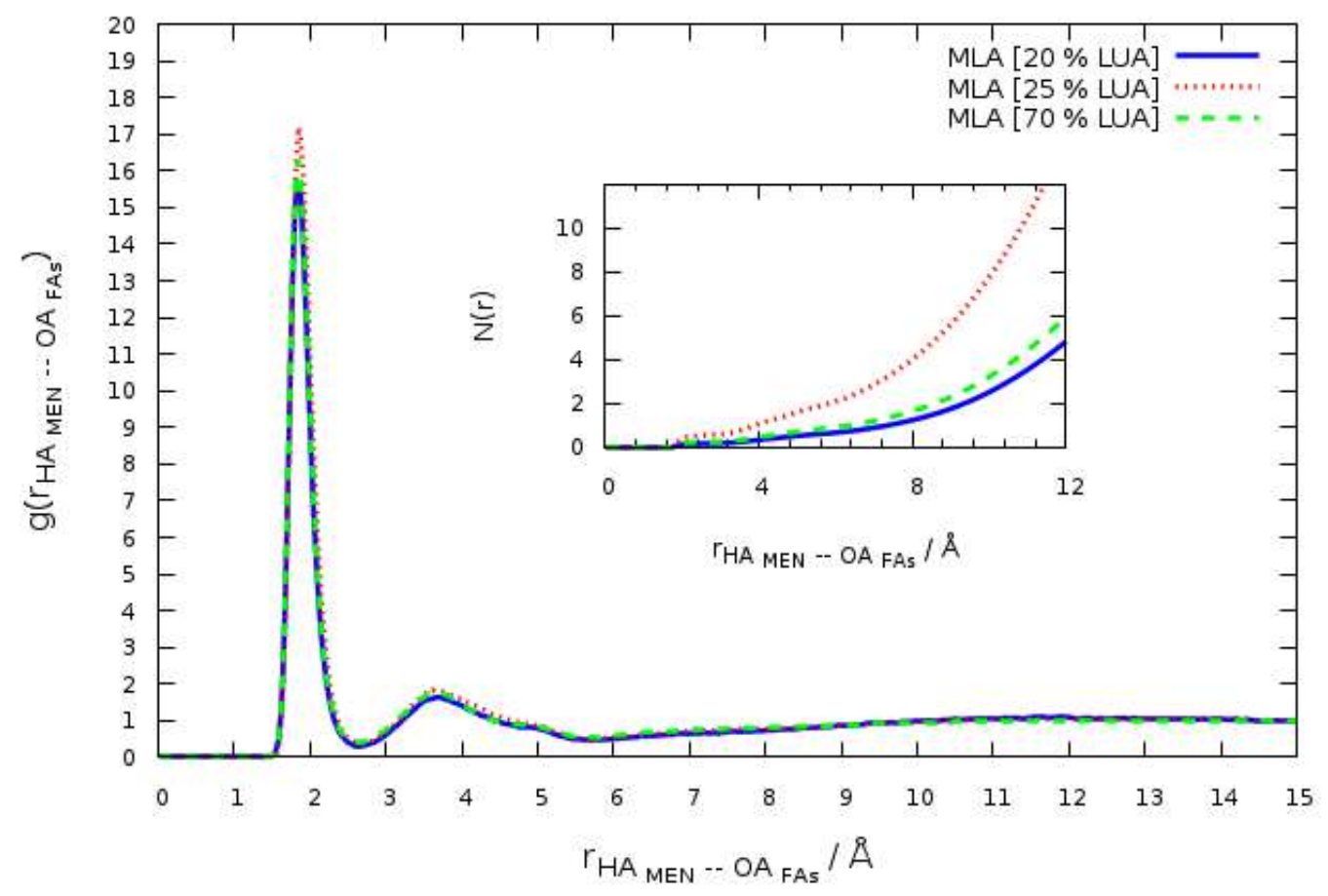

Fig. 2b. RDFs between HA atom on the menthol molecules and the OA atom of LUA in the binary mixtures with \% LUA $=20,25$, and 70 at $323 \mathrm{~K}$. 


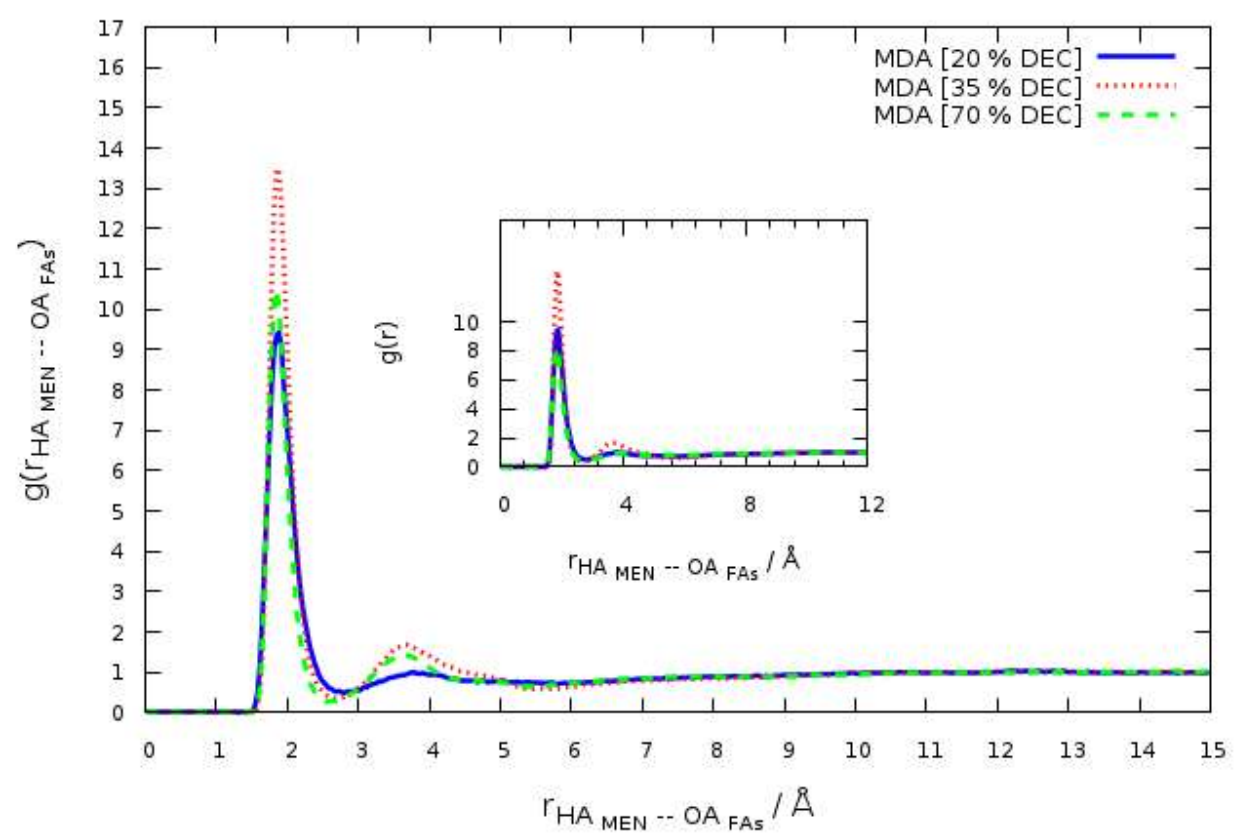

Fig. 2c. RDFs between HA atom on the menthol molecules and the OA atom of DEC in the binary mixtures with $\%$ DEC $=20,35$, and 70 at $323 \mathrm{~K}$.

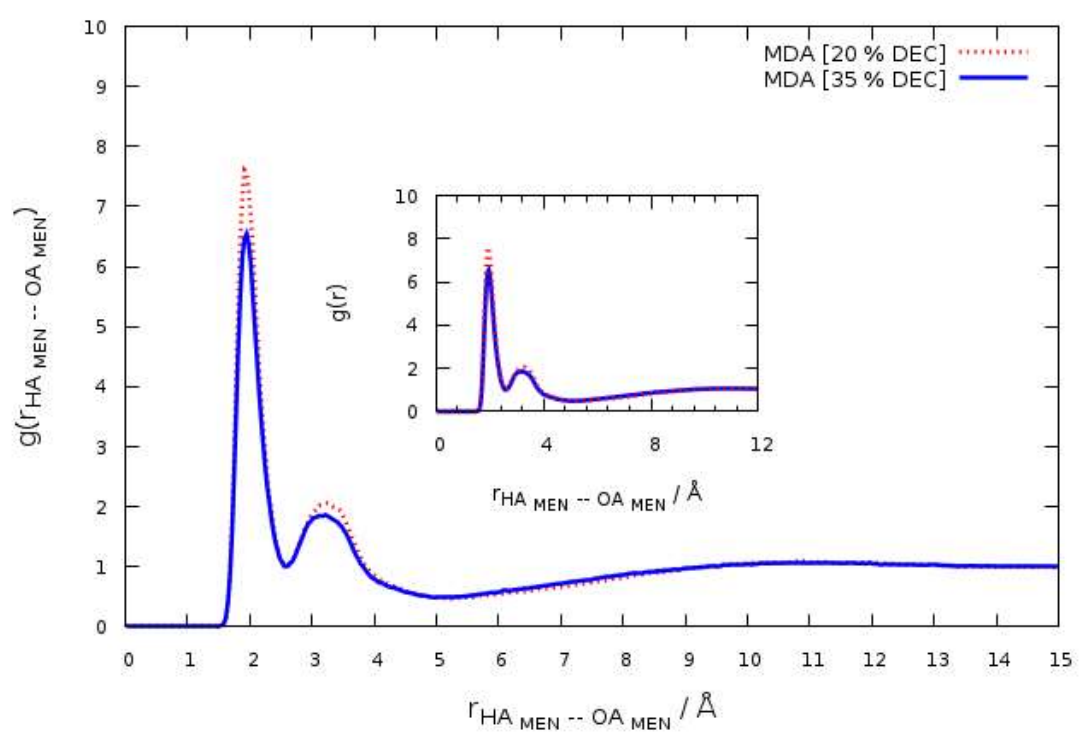

Fig. 2d. RDFs between menthol molecules, $g(r)_{\text {MEN -- MEN }}$, for the binary mixtures of MEN and DEC with \% DEC $=20$ and 35 at $323 \mathrm{~K}$. 


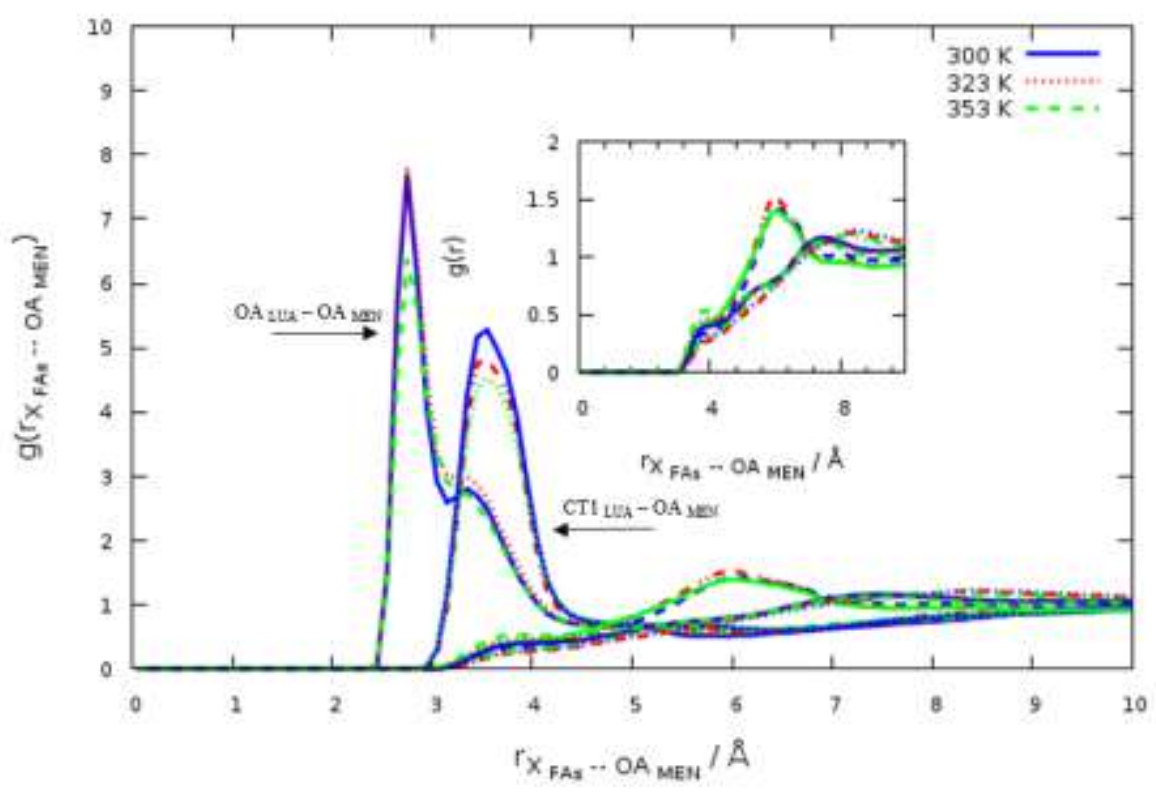

Fig. 2e. Site-site RDFs between atoms of the LUA molecules and the OA on the menthol molecule in the binary mixtures at $323 \mathrm{~K}$.

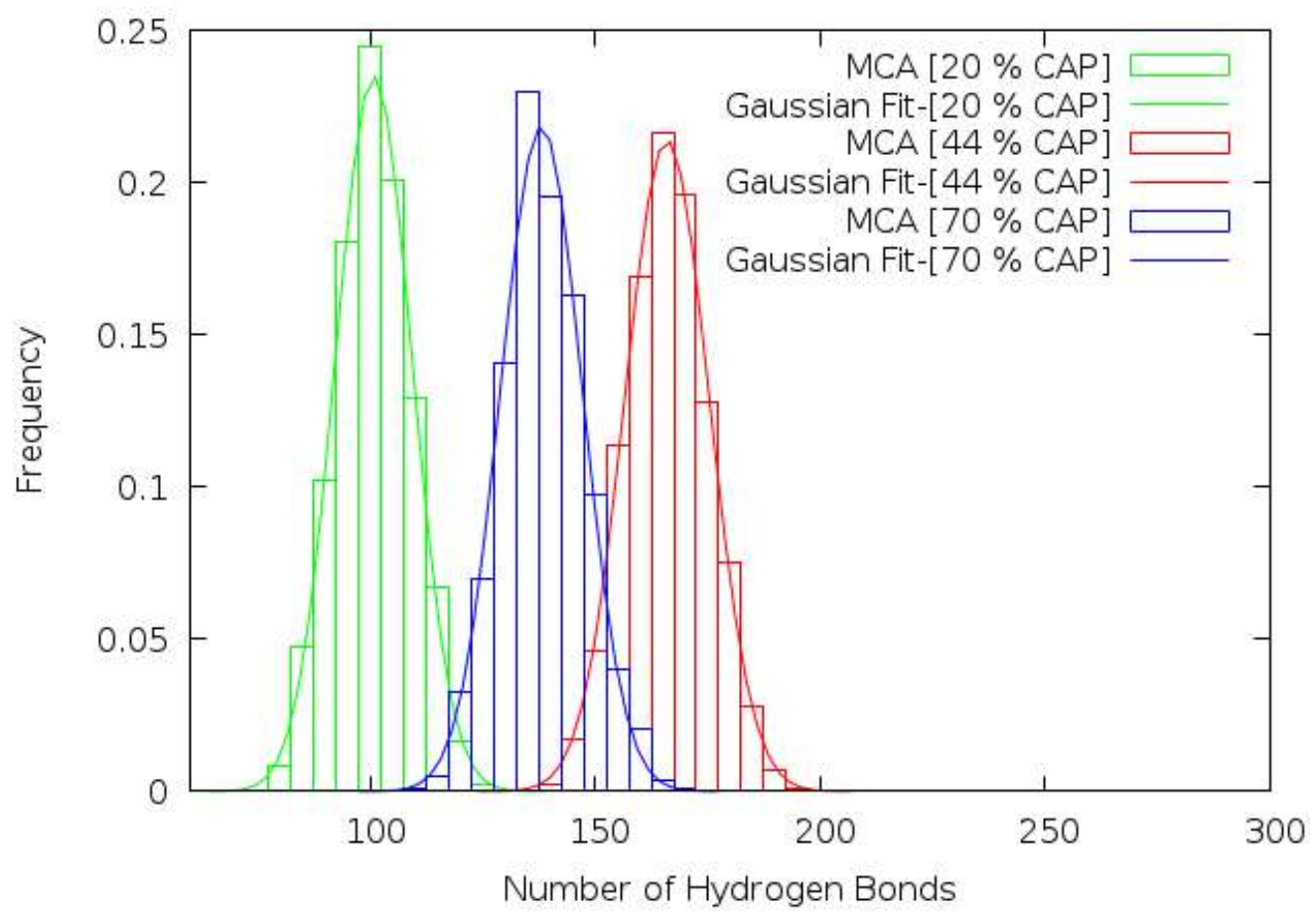

Fig. 5a. The distribution of the hydrogen bond between menthol and CAP molecules in the binary mixtures with \%CAP $=20,44$ and 70 at $323 \mathrm{~K}$. 


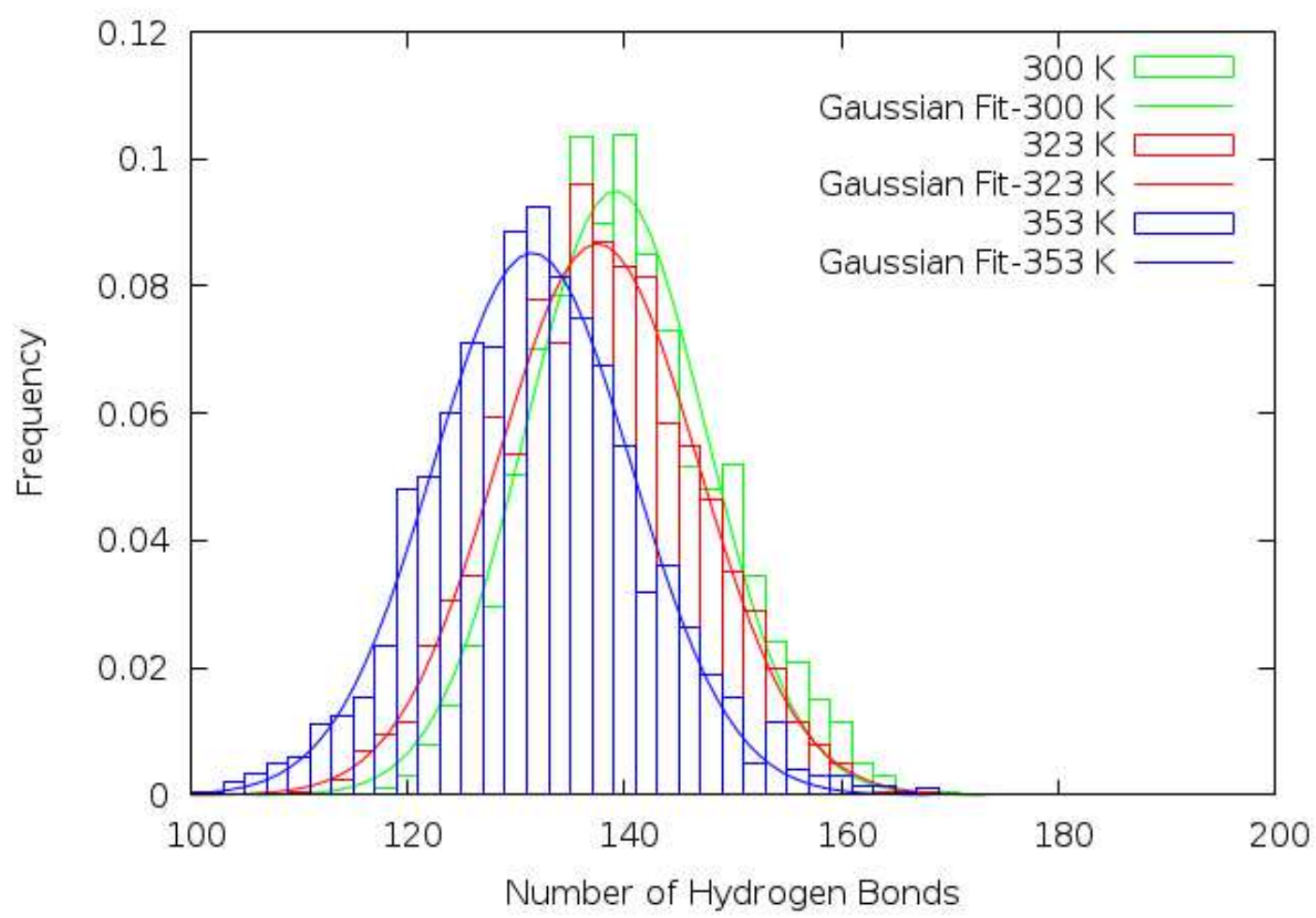

Fig. 5b The distribution of the hydrogen bond between menthol molecules in the binary mixture with \%LUA $=44$ at different temperatures.

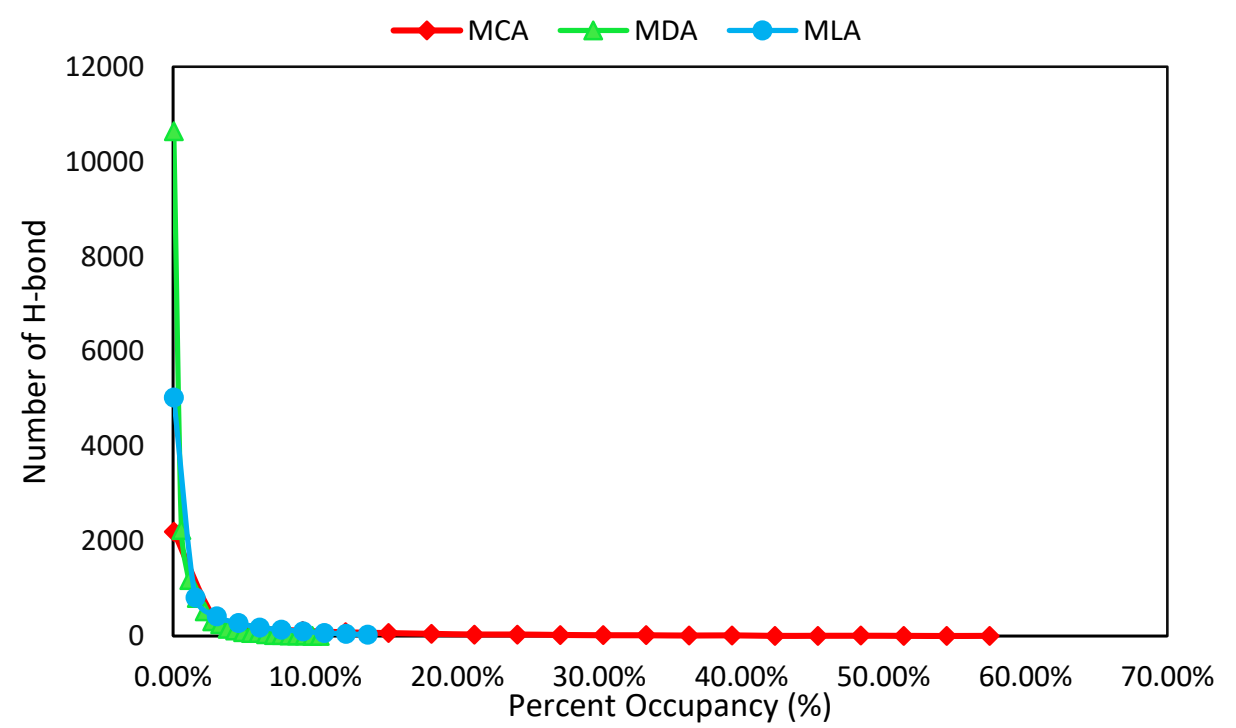

Fig. 6a. Hydrogen bond percent occupancies between the $\mathrm{H}$ atoms of MEN and the $\mathrm{O}$ atom of FAs in the binary mixture with a molar percentage of $70 \%$ at $323 \mathrm{~K}$. 


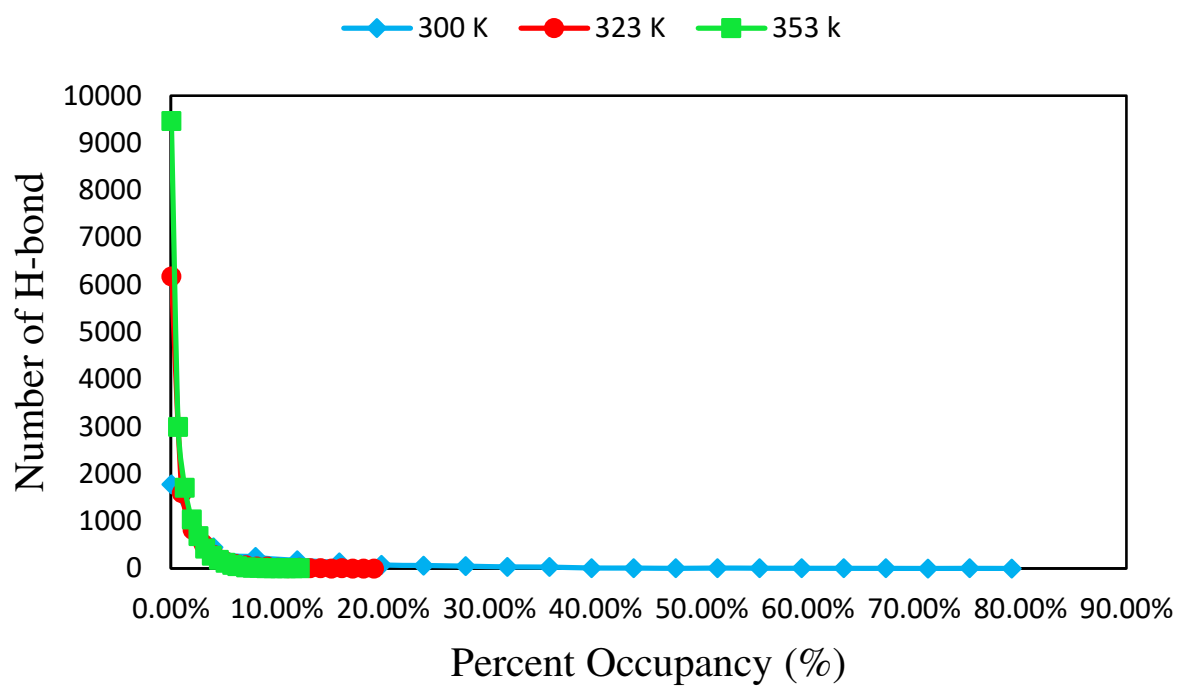

Fig. 6b. Hydrogen bond percent occupancies between the $\mathrm{H}$ atoms of MEN and the OA atom of LUA in the binary mixture with a molar percentage of $25 \%$ at different temperatures.

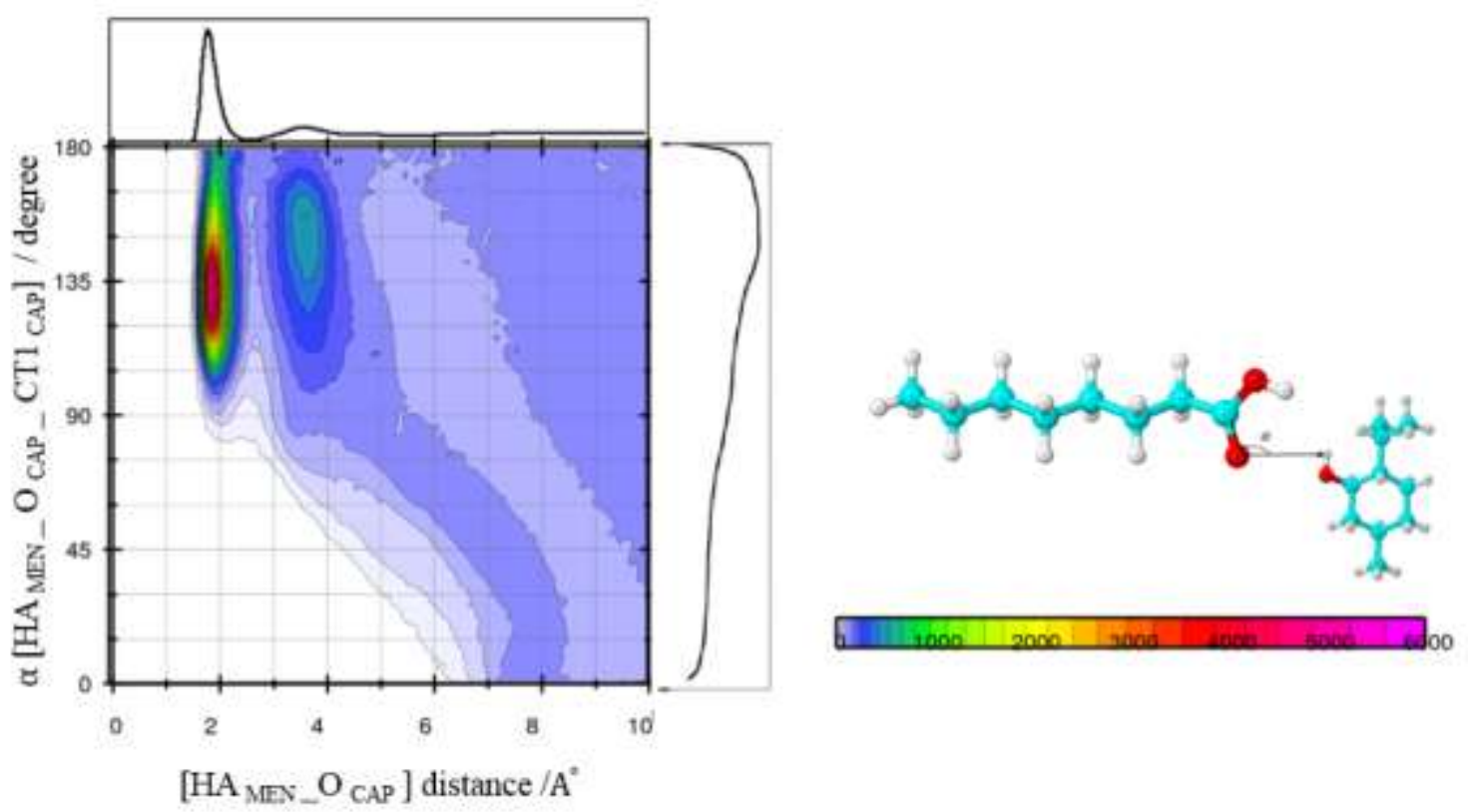

Fig. 8a. Combined radial/angular distribution functions for the HA MEN_O CAP distance and HA MEN_O CAP_CT1 CAP angle, in the binary mixtures with \%CAP $=70$ at $323 \mathrm{~K}$ 

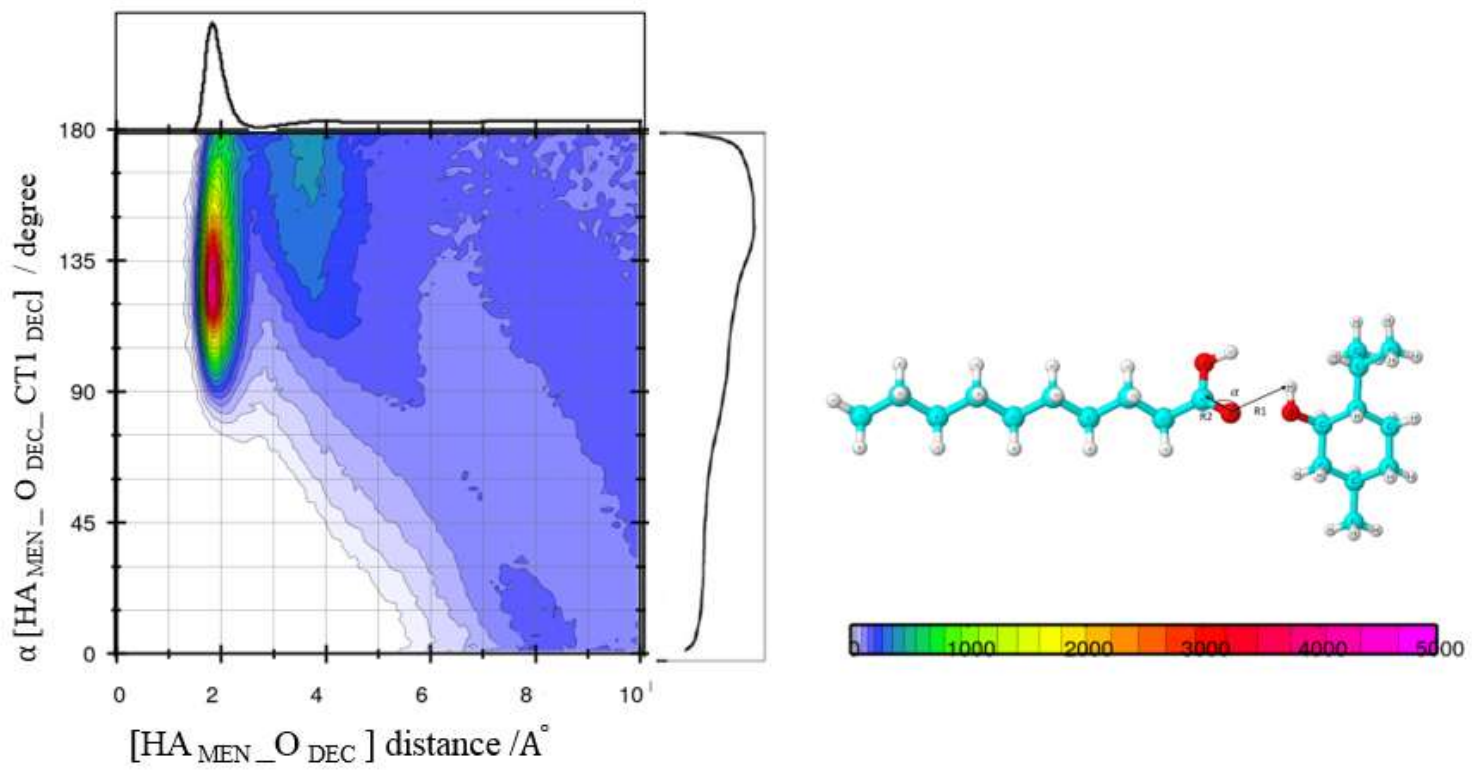

Fig. 8b. Combined radial/angular distribution functions for the HA MEN_O DEC distance and HA MEN_O DEC_CT1 DEC angle, in the binary mixtures with $\%$ DEC $=70$ at $323 \mathrm{~K}$.

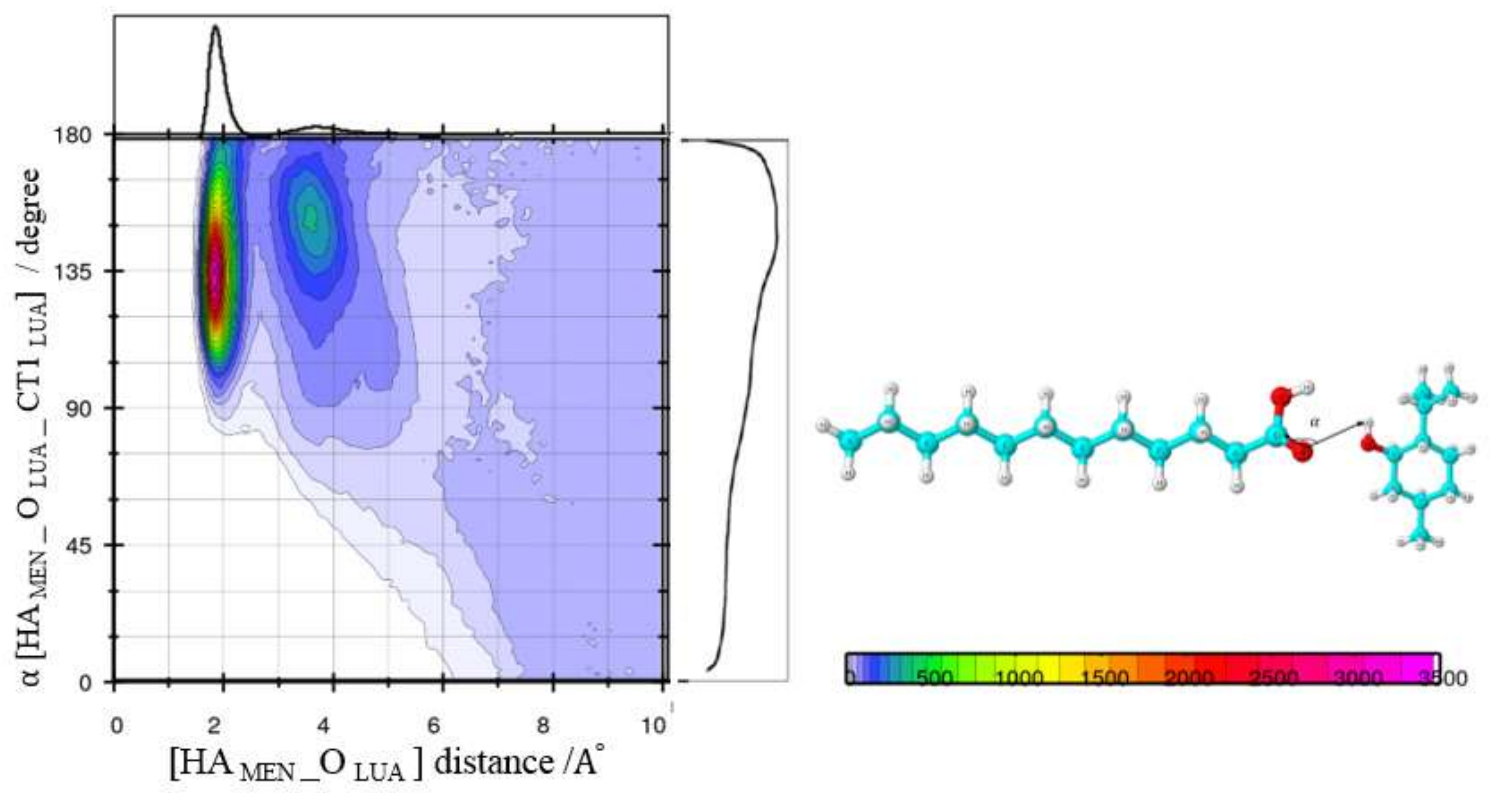

Fig. 8c. Combined radial/angular distribution functions for the HA MEN_O $\mathrm{O}_{\text {LUA }}$ distance and HA MEN_O LUA_CT1 LUA angle, in the binary mixtures with \%LUA $=70$ at $323 \mathrm{~K}$ 


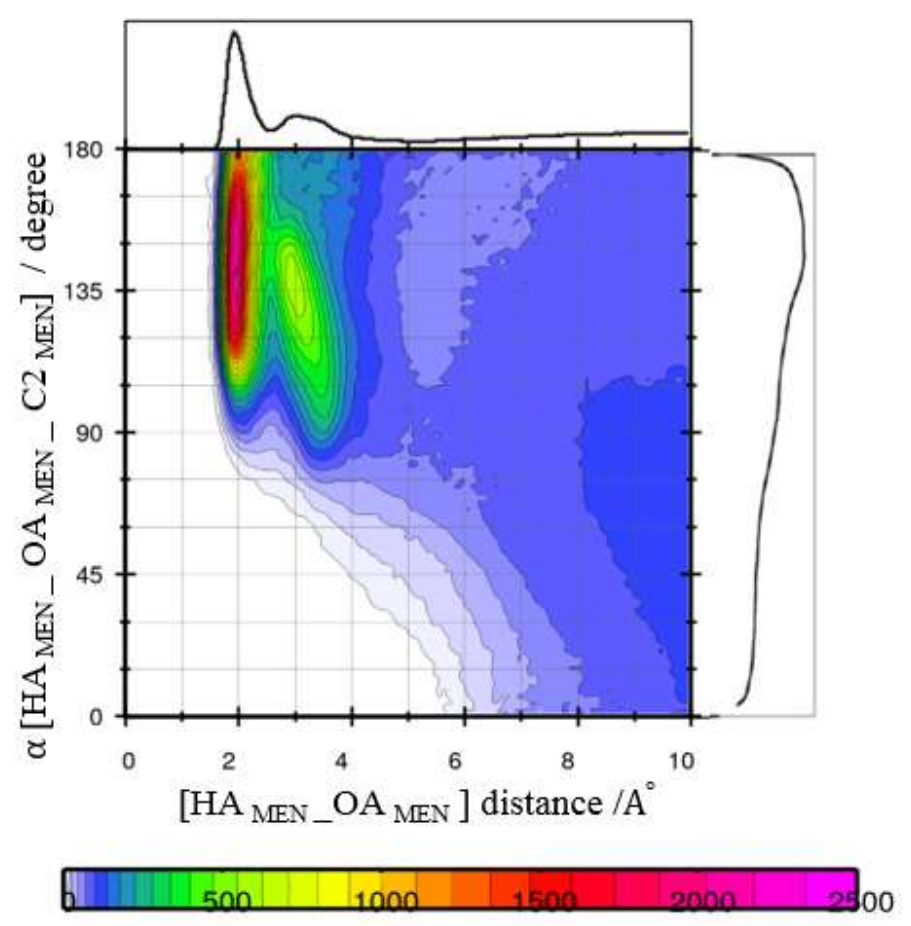

Fig. 8d. Combined radial/angular distribution functions for the HA MEN_OA MEN distance and HA MEN_OA MEN_C2 MEN angle, in the binary mixtures with $\% \mathrm{CAP}=44$ at $323 \mathrm{~K}$.

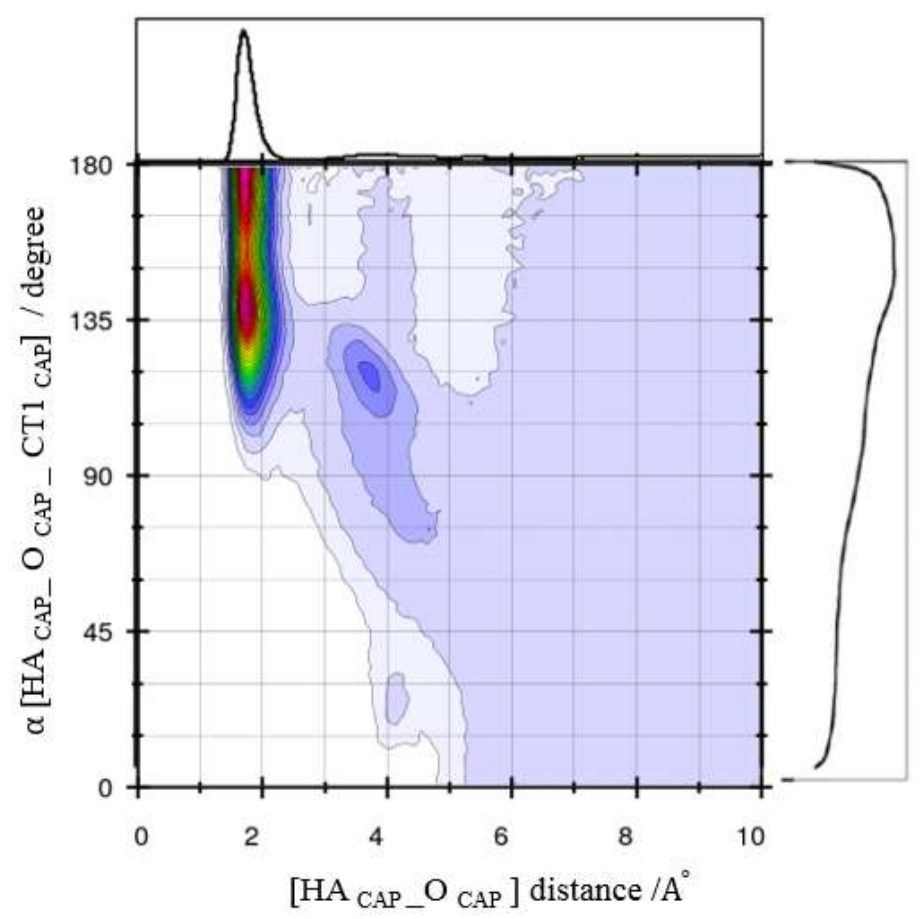

Fig. 8e. Combined radial/angular distribution functions for the HA CAP_O CAP distance and HA CAP_O CAP_CT1 CAP angle, in the binary mixtures with $\%$ CAP $=44$ at $323 \mathrm{~K}$ 


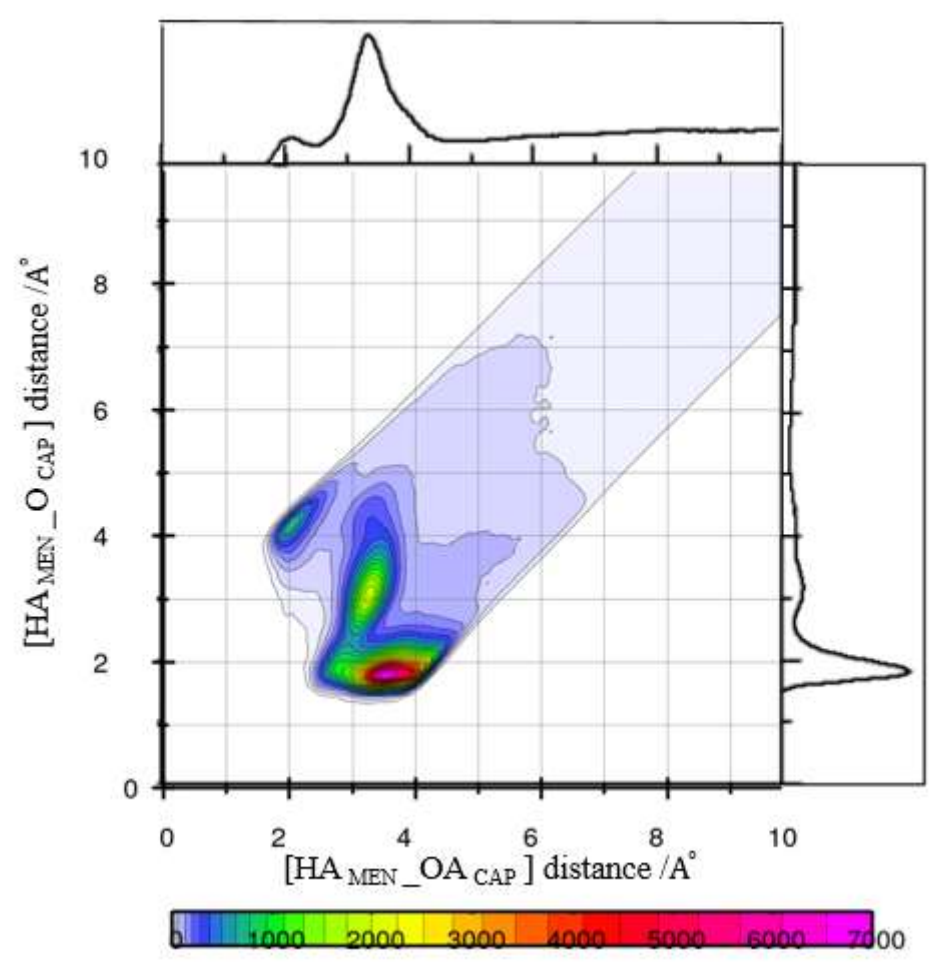

Fig. 9a. Combined radial/ radial distribution functions for the HA MEN _O ${ }_{\text {CAP }}$ distance and HA MEN _OA ${ }_{\text {MEN }}$ distance, in the binary mixtures with $\% \mathrm{CAP}=20$ at $323 \mathrm{~K}$.

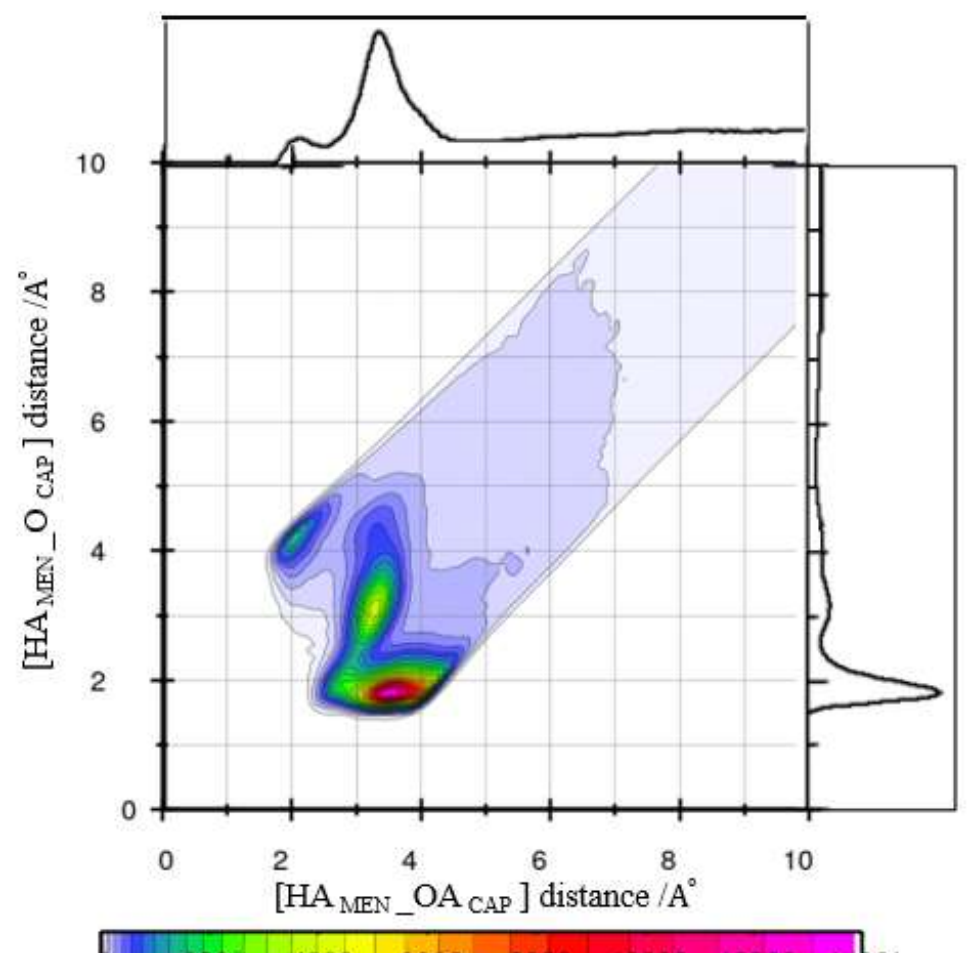

Fig. 9b. Combined radial/ radial distribution functions for the HA CAP_O ${ }_{\text {CAP }}$ distance and HA CAP _OA MEN distance, in the binary mixtures with $\% \mathrm{CAP}=44$ at $323 \mathrm{~K}$. 


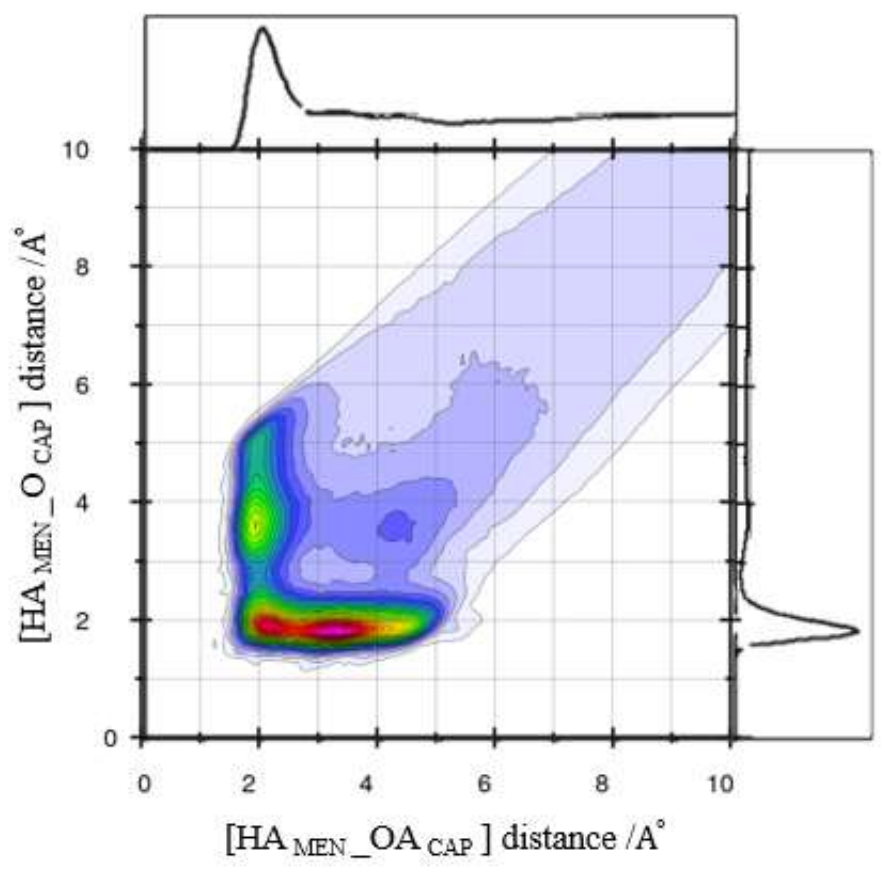

\begin{tabular}{|l|l|llllll}
\hline II & 500 & 1000 & 1500 & 2000 & 2500 & 3000 & 3000
\end{tabular}

Fig. 9c. Combined radial/ radial distribution functions for the HA CAP_O ${ }_{\text {CAP }}$ distance and HA CAP _OA MEN distance, in the binary mixtures with $\% \mathrm{CAP}=70$ at $323 \mathrm{~K}$.

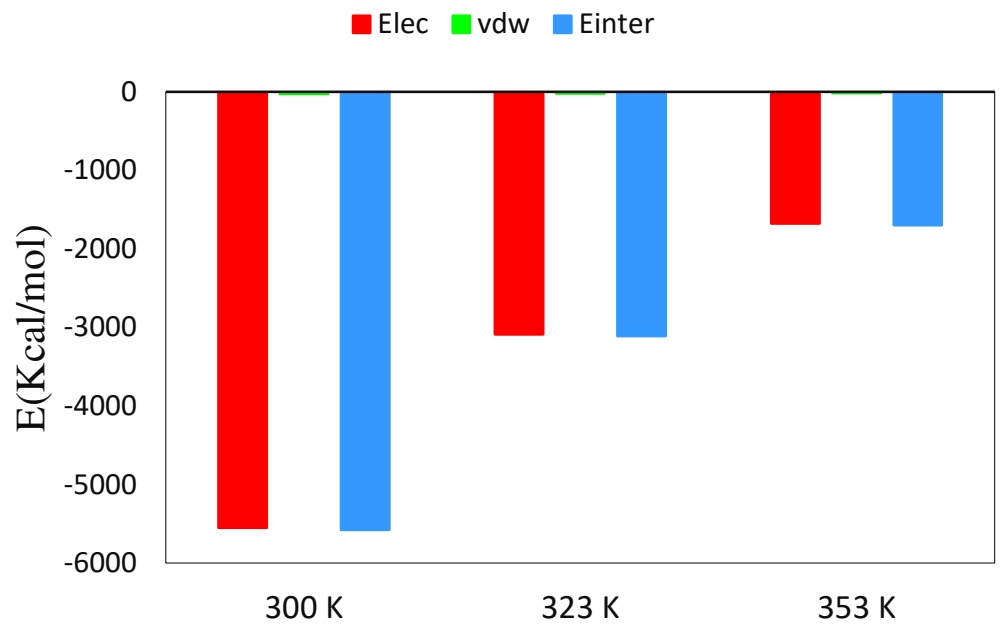

Fig. 10. Intermolecular interaction energy, $E_{\text {inter, }}$, Van der Waals, $E_{v d w}$, Electrostatic, $E_{\text {coul }}$, between MEN: MEN for the binary mixtures of menthol and fatty acid with \%LUA $=44$ at different temperatures. 


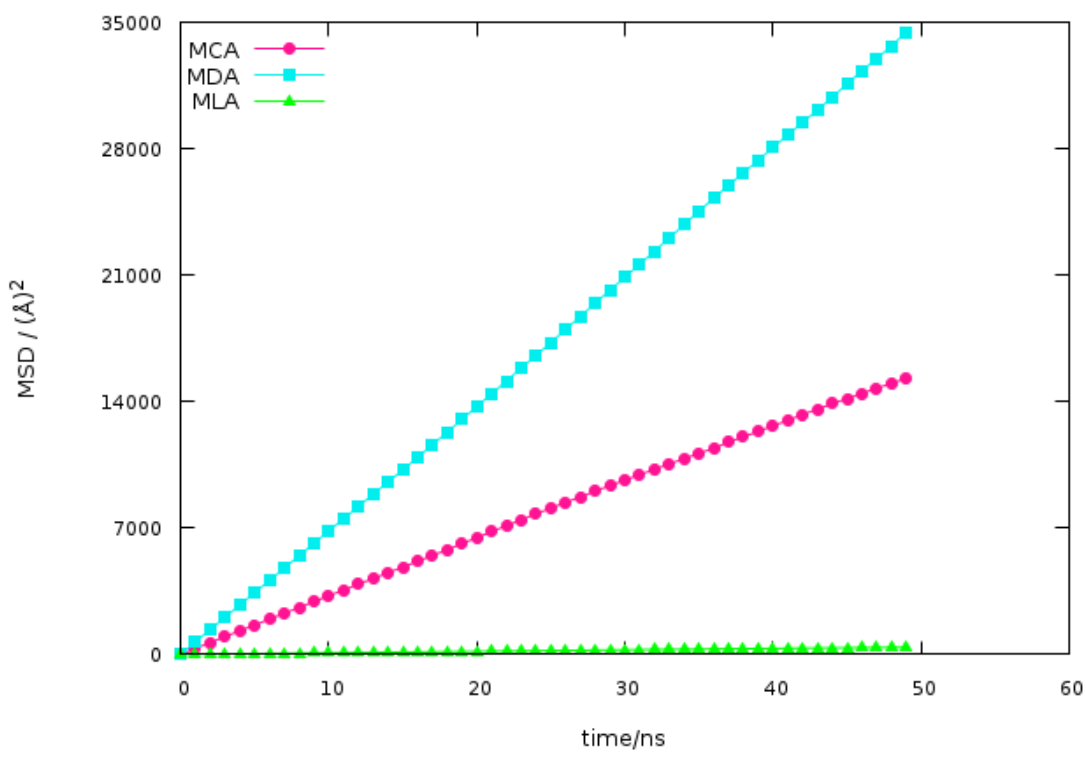

Fig. 11a. The MSDs of the MEN for the binary mixtures of menthol and fatty acid with $\% \mathrm{FAs}=70$ at $323 \mathrm{~K}$.

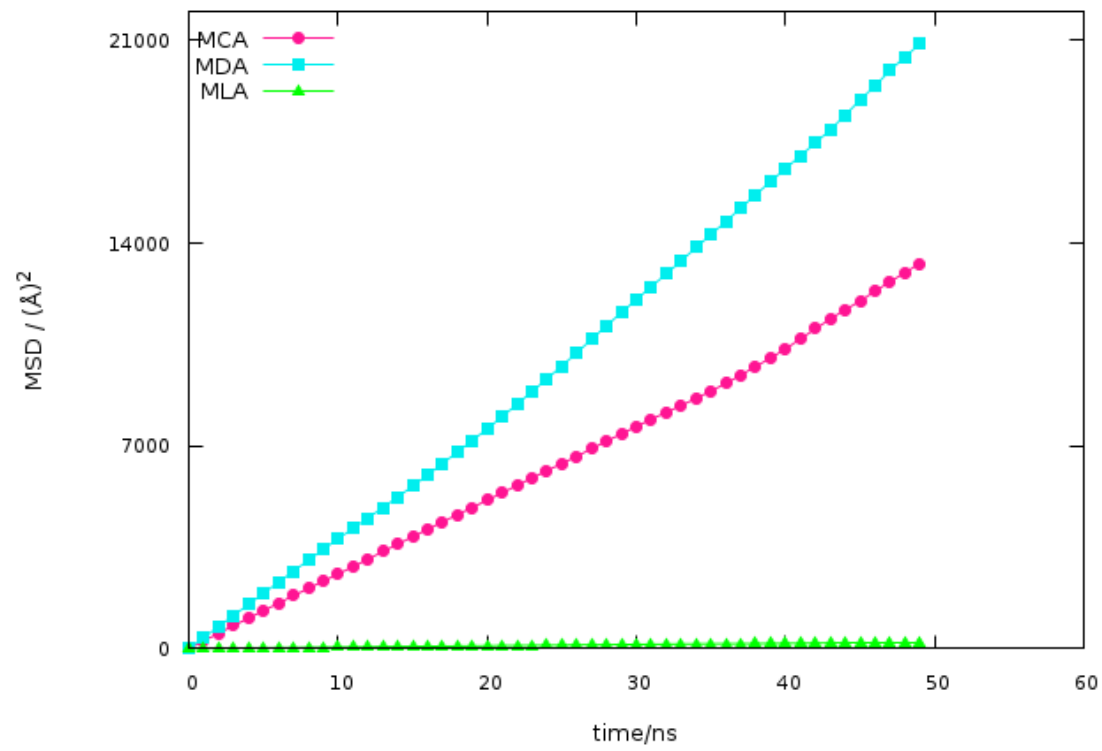

Fig. 11b. The MSDs of the FAs for the binary mixtures of menthol and fatty acid with $\% \mathrm{FAs}=70$ at $323 \mathrm{~K}$. 


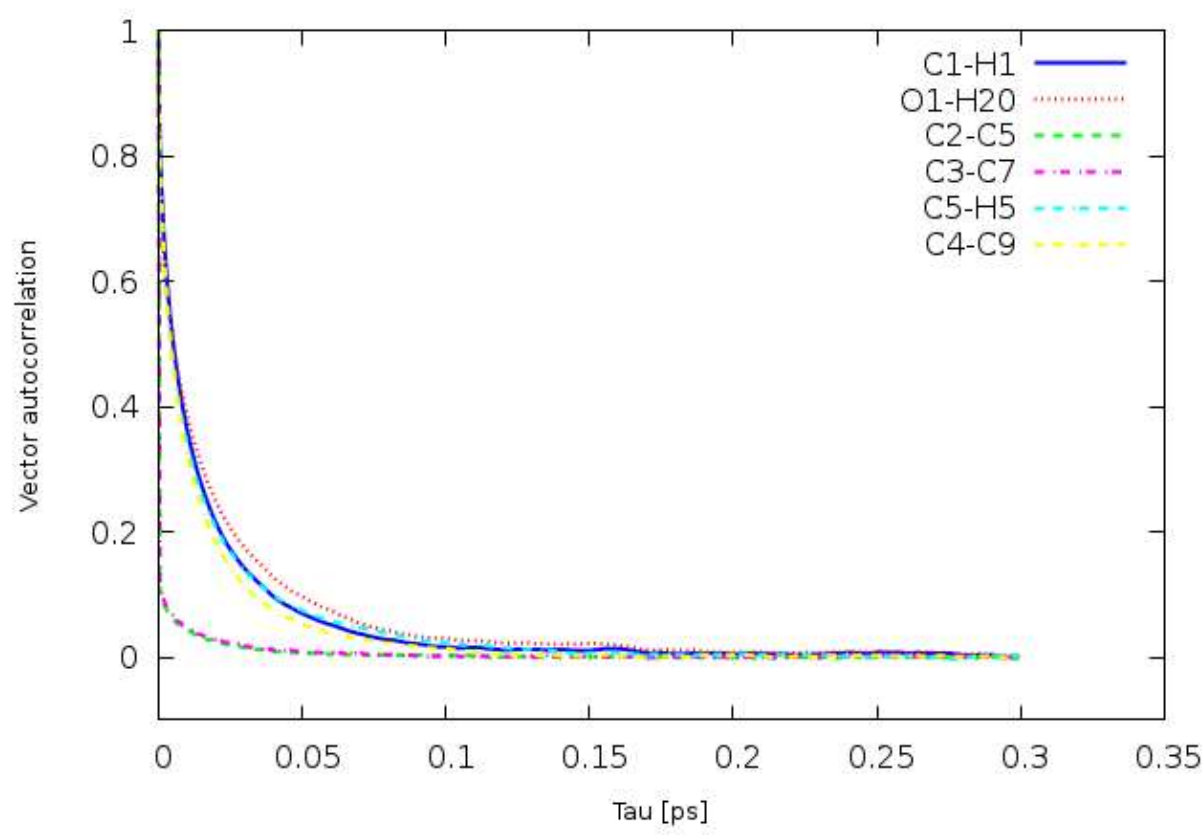

Fig. 12a. Vector reorientation dynamics for bond vectors of MEN in the binary mixture of MCA with \%CAP $=44$ at $323 \mathrm{~K}$.

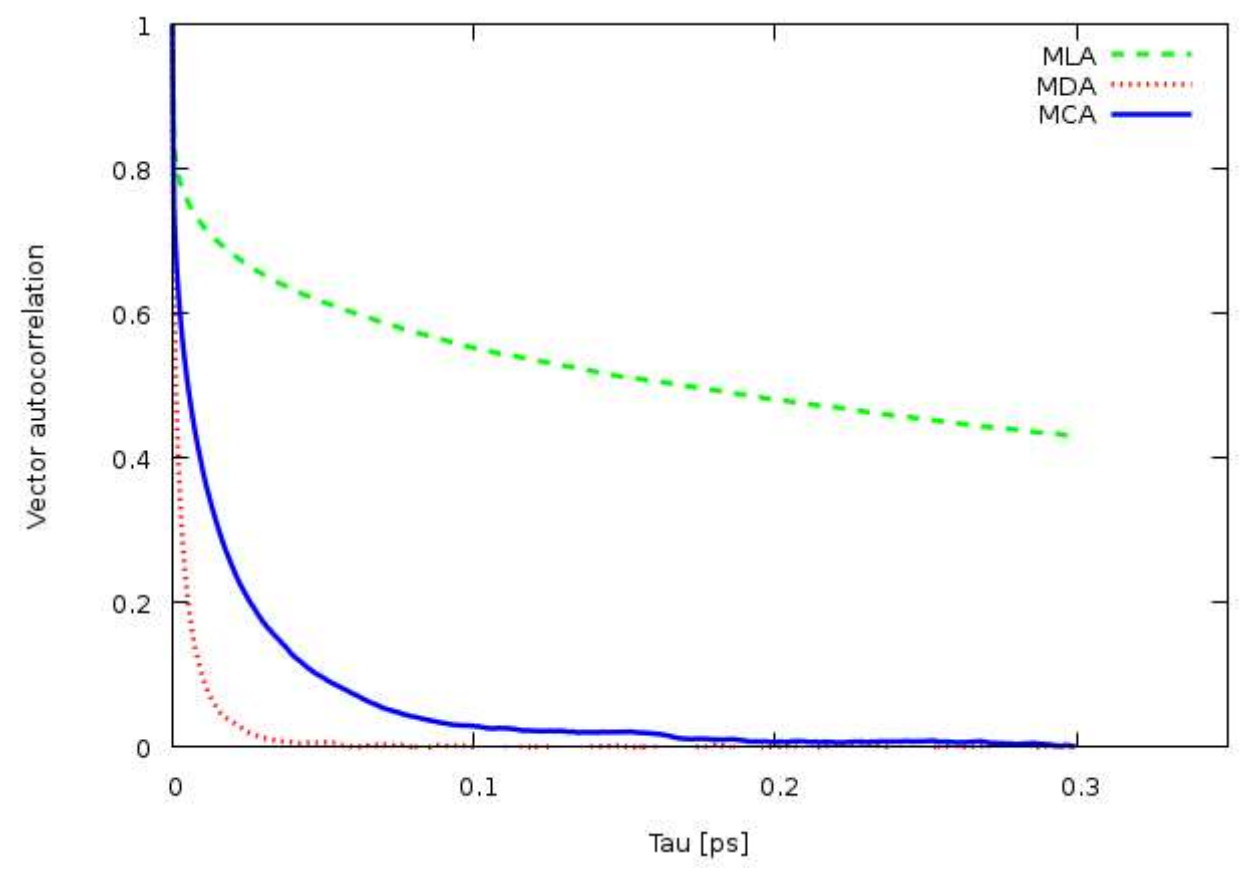

Fig. 12b. Vector reorientation dynamics for bond vectors (HA-OA) of MEN in the binary mixture of MCA with $\% \mathrm{FAs}=70$ at $323 \mathrm{~K}$. 


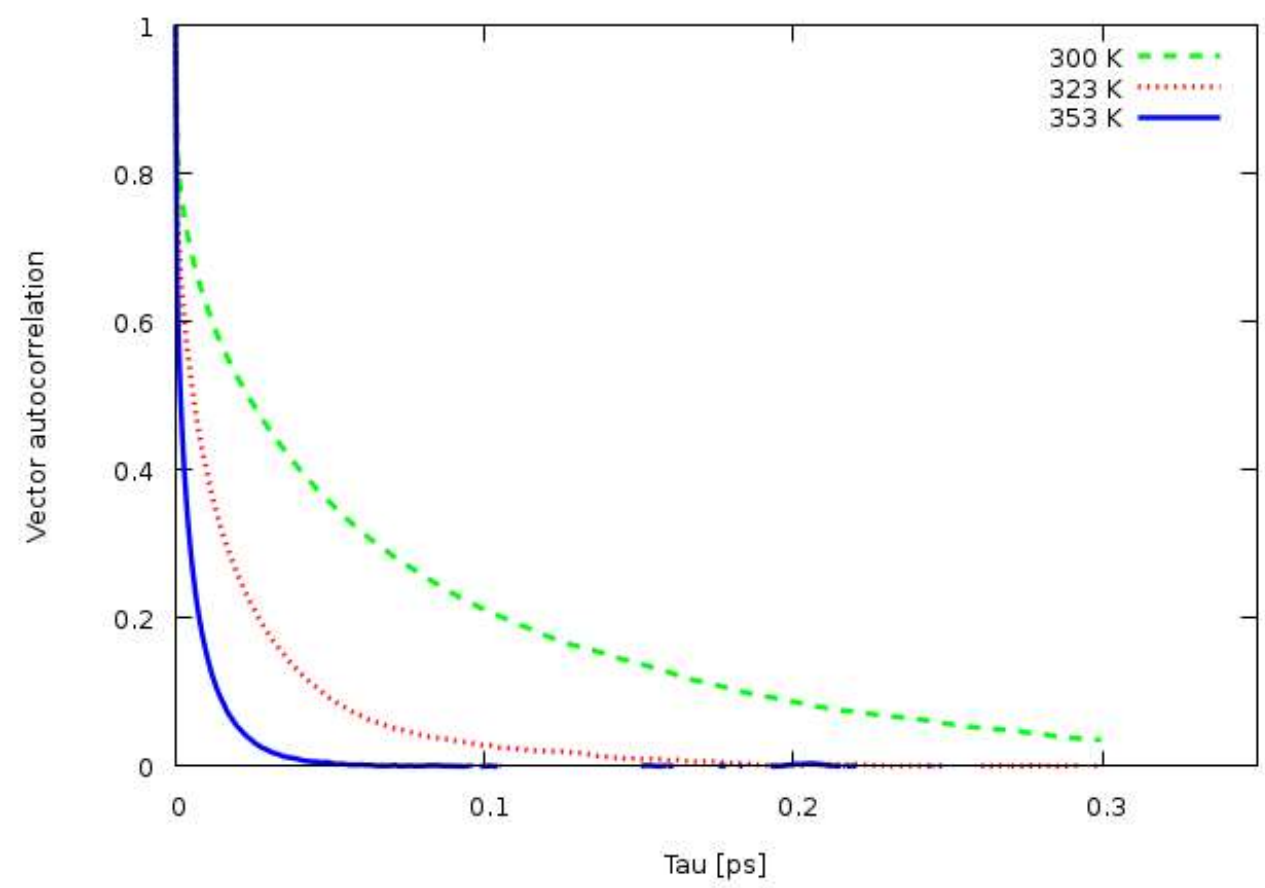

Fig. 12c. Vector reorientation dynamics for bond vectors (HA-OA) of MEN in the binary mixture of MLA with \% LUA $=25$ at different temperatures. 


\section{Supplementary Files}

This is a list of supplementary files associated with this preprint. Click to download.

- SupportingInformation.docx 